Article

\title{
Coumaroyl Flavonol Glycosides and More in Marketed Green Teas: An Intrinsic Value beyond Much-Lauded Catechins
}

\author{
Lorenzo Candela, Marialuisa Formato, Giuseppina Crescente, Simona Piccolella $\mathbb{D}$ and \\ Severina Pacifico * (D) \\ Department of Environmental Biological and Pharmaceutical Sciences and Technologies, University of \\ Campania “Luigi Vanvitelli”, Via Vivaldi 43, 81100 Caserta, Italy; lorenzocandela94@gmail.com (L.C.); \\ marialuisa.formato@unicampania.it (M.F.); giuseppina.crescente@unicampania.it (G.C.); \\ simona.piccolella@unicampania.it (S.P.) \\ * Correspondence: severina.pacifico@unicampania.it
}

Received: 30 March 2020; Accepted: 9 April 2020; Published: 11 April 2020

\begin{abstract}
Marketed green teas (GTs) can highly vary in their chemical composition, due to different origins, processing methods, and a lack of standardization of GT-based products. Consequently, biological activities become difficult to correlate to the presence/content of certain constituents. Herein, ultra-high-performance liquid chromatography (UHPLC) combined with high-resolution tandem mass spectrometry (HR MS/MS) was successfully applied to six commercial GT products, extracted by ethanol sonication, to disclose their polyphenol profile beyond the well-known catechins. The relative abundance of each class of metabolites was correlated to antiradical and antilipoperoxidant data through hierarchical clustering analysis, since it reasonably affects the beneficial properties of the product that reaches the consumer. The thiobarbituric acid reactive substances (TBARS) assay demonstrated that GT extracts effectively counteracted the UV-induced lipoperoxidation of hemp oil, which is highly rich in Polyunsaturated Fatty Acids (PUFAs), and therefore highly unstable. The Relative Antioxidant Capacity Index (RACI) comprehensively emphasized that gunpower and blend in filter GTs appeared to be the less active matrices, and except for a GT-based supplement, the Sencha GT, which was particularly rich in flavonol glycosides, was the most active, followed by Bancha GT.
\end{abstract}

Keywords: marketed green tea; ultrasound assisted maceration; UHPLC-HRMS metabolic profile; coumaroyl flavonol glycosides

\section{Introduction}

Tea from leaves of Camellia sinensis (Theaceae family) is one of the most popular beverages, whose consumption worldwide has a long history. It has always played a leading role in Chinese history and in China's relations with near and far foreign cultures. It has long been considered a luxury product, highly sought after by all the people who acquired its stimulating taste [1]. The growing popularity of this beverage with valuable medicinal properties quickly spread to other East Asian cultures, especially Japan. The tea introduction in Europe defined its approval, fame, and its big business so much so that its global market was valued at nearly 50 billion U.S. dollars in 2017 and is expected to rise to over 73 billion dollars by 2024 [2].

The huge tea variety on the market is the result of different processing methods, in which withering, rolling, oxidation, and drying are the common steps [3]. Dark teas undergo high oxidation levels, whereas green tea completely skips oxidation, avoiding the browning process and thus preserving the green color of the leaf matrix and its richness in bioactive polyphenols. Catechins comprise 
$80-90 \%$ of flavonoid content [4], and they are especially pointed out as green tea (GT) catechins, as flavan-3-ols in GT mostly show a galloyl moiety on the C-3 carbon of catechin core or a pyrogallol B-ring. Catechins are nutraceutical compounds, exerting a preventive efficacy in offsetting oxidant species over-genesis in normal cells, and for their ability to halt or reverse oxidative stress-related diseases [5-7]. In this framework, multiple scientific evidences support the catechin-related health benefits of green tea consumption that include blood flow improvement, cardiovascular disease prevention, toxins elimination, and resistance enhancement to various diseases $[8,9]$. Cancer prevention with green tea was also recently reviewed; green tea, beyond the ability to delay cancer onset and to reduce its incidence, was reported as a useful strategy in secondary and tertiary cancer prevention [10]. Furthermore, cardiometabolic diseases, such as the ongoing metabolic syndrome, dyslipidemia, and diabetes, are reported to be beneficially alleviated by green tea [11]. These findings appeared to promote the therapeutic implementation of green tea [12]. Thus, in the great tea world, green tea has become very widespread over the last decades, and among the different Chinese and Japanese green tea leaves currently available on the market, also thanks to food sector operators and mass media advertisement, various supplements enriched in green tea catechins were prepared. Indeed, scientific data are uncertainly translated into meaningful benefits [13], which is mainly due to the instability and low bioavailability of green tea compounds. Furthermore, food processing such as pasteurization, sterilization, and storage could alter the structure of polyphenols [14], which show a diversity that is far beyond the well-known and abundant catechins. In fact, green tea contains, as other tea varieties, flavonol 3-O-glycosides $[15,16]$, as well as other typical compounds such as theophylline, theanine, and theobromine [17]. Nevertheless, the content of the other polyphenolic components has long been disregarded, whereas great efforts have been made to prepare catechin-based supplements that are particularly enriched in epigallocatechin gallate (EGCG), which, among the other catechins, appears to be the most reactive substance. Indeed, although EGCG in a dose range level equal to $800-1600 \mathrm{mg}$ seems to be well tolerated [18,19], adverse hepatotoxic effects, probably due to a GT catechins abuse, pose health concerns [20]. The European Food Safety Authority (EFSA), reviewing the conclusions of several studies aimed at evaluating the effects of EGCG on the liver, concluded that if the green tea beverage intake is generally safe, some precautions must be taken toward supplements, because many of them bring doses of catechins that can cause liver health problems [21,22]. In reality, the great variability of the preparation methods and the composition of the supplements means that the amount of catechins in these products can differ considerably between one product and another. In this context, Hu et al. [23], reviewing the scientific literature, highlighted that the large part of the toxicological studies lacks a detailed chemical characterization of the tested material. The lack of standardization (or the not full understanding) of the composition of the green tea preparations in marketed products adds further complexity to the risk assessment.

Herein, in order to compare the chemical composition of Camellia sinensis leaves of different origins in marketed products (obtained with different processing methods) and to mainly verify their metabolic profile in polyphenol compounds, beyond catechins and further antioxidant activity, six different green tea-based materials underwent ultrasound-assisted maceration (UAM) using ethanol as extracting solvent. In particular, Chinese gunpowder tea, three Japanese teas (Sencha, Bancha and Matcha), a commercial preparation in a filter bag, and one green tea catechin-enriched supplement were investigated. Gunpowder tea, a form of Chinese tea in which each leaf has been rolled into a small round pellet, and Japanese green teas, Sencha, Bancha, and Matcha teas were selected. Sencha is made from cultivated tea bushes of Camellia sinensis var. sinensis and it is commonly from the upper leaves and buds, whereas the lower more mature leaves are usually used for Bancha. Instead, Matcha is an unfermented tea that is steamed, dried, and ground into a milled green tea powder. The alcoholic extracts obtained were analyzed for their metabolomic profile by applying UHPLC HR MS/MS techniques. The extracts obtained were used for a preliminary bioactivity screening using DPPH (2,2'-diphenyl-1-picrylhydrazyl), ABTS (2,2'-azino-bis(3-ethylbenzothiazoline-6-sulfonic acid)), ORAC (oxygen radicals absorbance capacity), and thiobarbituric acid reactive substances (TBARS) assays. 


\section{Results and Discussion}

\subsection{Chemical Composition of Alcoholic Extracts from Commercialized Green Tea-Based Materials}

The catechin content of the selected green tea types were compared to that of a green tea catechin-based supplement, whereas the diversity in other phenolic, polyphenolic, and non-phenolic compounds was also evaluated. To this purpose, taking into account that ethanol is a good solvent for polyphenol extraction [24], also for higher molecular weight flavanols, alcoholic extracts were prepared by means of ultrasound-assisted maceration [25]. Previous investigation on black tea highlighted that the use of ultrasonic intensification led to an increase in polyphenols by approximately $15 \%$ in respect to conventional maceration [26]. Furthermore, it was observed that compared to the conventional shaking extraction methods, ultrasonic-assisted maceration on green tea leaves takes advantage of less extraction time and lower temperature [27]. The extraction scheme and the simple applied workflow are depicted in Figure 1.

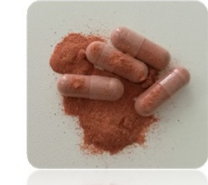

GTC supplement

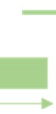

EtOH extracts

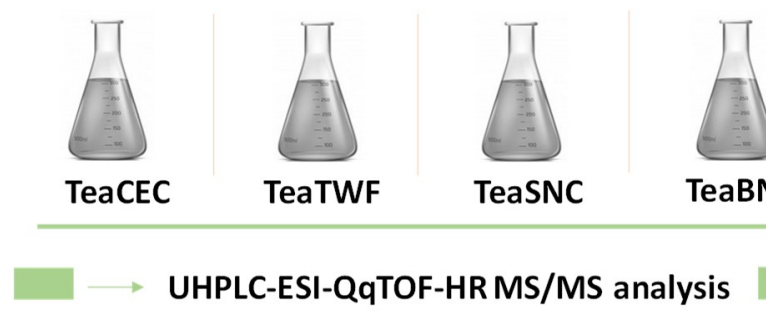

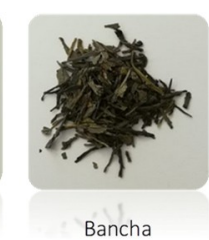

Ultrasound Assisted Maceration (UAM)

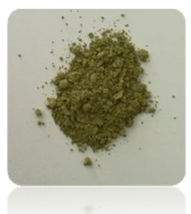

Matcha

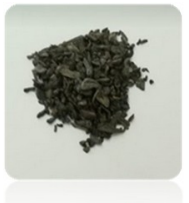

Gunpowder

Figure 1. Extraction scheme applied on selected green tea matrices.

The prepared extracts were investigated by means of ultra-high-performance liquid chromatography coupled with high-resolution mass spectrometry (UHPLC-HRMS). The total ion current chromatograms of extracts were mostly superimposable (Figure S1). As expected, flavanol compounds were the most representative, whereas flavonol substances, glycosylated and/or acylated, were also present in all the extracts but that obtained through ultrasound-assisted maceration on the green tea catechins-enriched supplement (TeaCEC). This latter almost completely lacked the saccharide and the great part of the flavonolic components. TeaCEC also did not contain theanine (2, Table 1), a non-protein amino acid contributing to the favorable taste $[28,29]$, which was in a range of $0.34-0.57 \%$ in the other prepared extracts. The presence of this unique free amino acid was slightly more abundant in gunpowder green tea extract (TeaGNP, 0.54\%) and Matcha tea extracts (TeaMTC, $0.57 \%$ ). The molecule was tentatively identified on the basis of the deprotonated molecular ion at $m / z 173.0932$ and the fragment ion at $m / z$ 155.0836, deriving from the neutral loss of a water molecule. The metabolites 1, 4, and 5 were saccharides (Figure S2, Table 1). In particular, the metabolite 1, showing the ion $[\mathrm{M}-\mathrm{H}]^{-}$at $m / z$ 311.0992, dissociated by supplying the ion at $m / z 179.0560$, due to a hexose unit, through the neutral loss of $132.0424 \mathrm{Da}$. This latter was according to a pentose residue. The presence of a pentose binding a hexose is also confirmed by the ion at $m / z 161.0453$. The metabolite 4 is putatively a trihexoside (e.g., raffinose). The ion $[\mathrm{M}-\mathrm{H}]^{-}$at $m / z 503.1637$ underwent the loss of two $\mathrm{C}_{6} \mathrm{H}_{10} \mathrm{O}_{5}$ moieties to provide the ions at $m / z 341.1097$ and 179.0558 . The ion at $m / z 341.1097$ gave rise by the loss of two $\mathrm{C}_{2} \mathrm{H}_{4} \mathrm{O}_{2}$ units the fragment ions at $m / z 281.0891$ and 221.0667 (Figure S2). 
Compound 5 was putatively a dihexose or, as hypothesized based on TOF-MS ${ }^{2}$ spectrum, a mixture of dihexoses. In fact, it was observed that when sucrose co-eluted with turanose, fragment ions at $m / z$ 143, 131, and 101 are detectable [30]. Both the disaccharides were previously reported as constituents of tea leaves and their abundance, together with that of maltose, cellobiose, and trehalose, appeared to be related to the tea plant growing condition as a response to cold stress [31].

Compounds 3 and $\mathbf{6}$ were tentatively identified as quinic acid and gallic acid, respectively, whereas the TOF-MS/MS spectrum of compound 7 was in accordance with theogallin, a depside of gallic acid and quinic one. Gallic acid and theogallin are the most abundant simple phenols in tea, and their relative content strongly depends on fermentation processes that favor the breakdown of the ester linkage, enhancing the gallic acid amount [32]. Indeed, gallic acid was found to be significantly released after enzymatic degradation during the fermentation process also from gallocatechin constituents [33]. Theogallin was suggested to slow down cognitive decline, improving brain health [34].

The metabolites $\mathbf{8 - 1 1}, \mathbf{1 4}, \mathbf{1 5}, \mathbf{1 7}, \mathbf{2 3}, \mathbf{2 6}, \mathbf{3 4}$, and 35 belong to the class of catechins (Table 2) and were identified as such on the basis of some of their characteristic peaks. Among the latter, the fragment ion at $m / z$ 125.02, corresponding to the ring A of the flavanolic nucleus [35], is produced following the HRF (heterocyclic ring fission) reaction. The fragment ion, common to all catechins, represents the base peak of molecules $\mathbf{8}$ and 10, which were identified on the basis of mass spectrometric data as gallocatechin isomers (Figure S3). Both the isomers were also characterized by the ions at $\mathrm{m} / \mathrm{z} 167.0356(3)$ and 137.0251(48), which are produced by RDA (retro Diels-Alder) reaction. The two deprotonated ions at $\mathrm{m} / \mathrm{z} 289.0722$ for compounds 11 and 14, eluted at different retention times, were in accordance with catechin and epicatechin diastereoisomers (Figure 2). The TOF-MS/MS spectra of both the molecules showed product ions at $m / z 245.0825$ (30) by carbon dioxide loss and A-ring cleavage, and further ethenone loss to achieve ions at $m / z$ 203.0719(23). The characteristic ions formed by reactions as HRF (at $m / z 125.0248(7)$ ) and RDA (at $m / z$ 137.0251(0) and 151.0407(5)) were also observed. The base peak at $m / z$ 109.0300(299) could be due to deprotonated catecholic moiety (B ring), whose loss as a neutral residue instead favored the formation of the fragment ion at $m / z$ 179.0353(58). A benzofuran-forming fission (BFF) reaction provided the ion at $\mathrm{m} / \mathrm{z} 123.0457(6)$.

A)

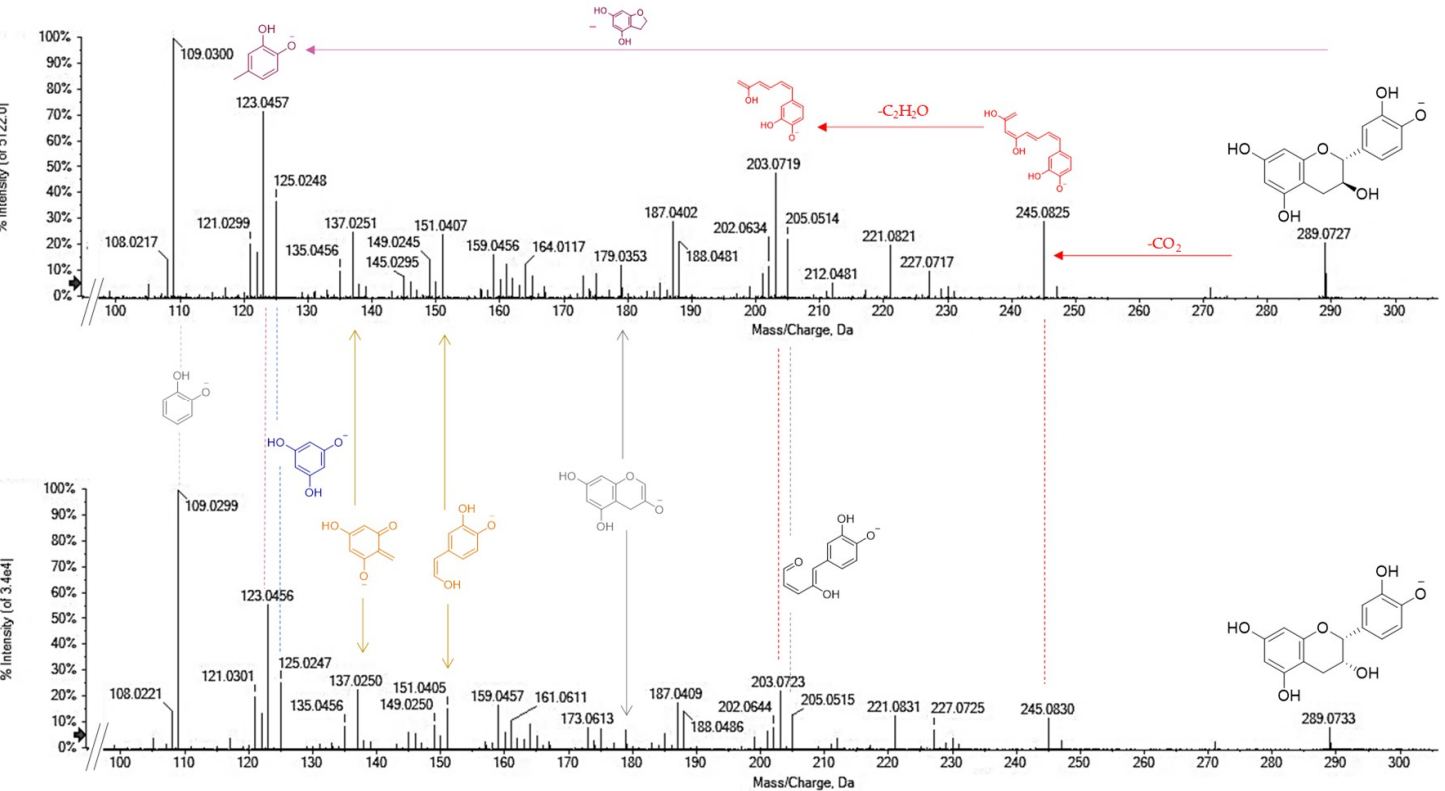

Figure 2. TOF-MS/MS spectra of $[\mathrm{M}-\mathrm{H}]^{-}$ion of compounds (A) 11 and (B) 14. The structure of the main fragment ions is highlighted. The relative abundance of the ions $\left[\mathrm{M}-\mathrm{H}-\mathrm{CO}_{2}\right]^{-}$and $[\mathrm{M}-$ $\left.\mathrm{H}-\mathrm{CO}_{2}-\mathrm{C}_{2} \mathrm{H}_{2} \mathrm{O}\right]^{-}$, as well as of ions from benzofuran-forming fission (BFF, in purple), heterocyclic ring fission (HRF, in blue), and retro Diels-Alder (RDA, in light orange) reactions allowed the geometrical isomers to be distinguished. 
Compound 9 was tentatively identified as (epi)gallocatechin dimer. The ion $[\mathrm{M}-\mathrm{H}]^{-}$at $m / z$ 609.1265 was according to the $\mathrm{C}_{30} \mathrm{H}_{26} \mathrm{O}_{14}$ molecular formula. The neutral loss of 126.0344 , due to the A-ring of a monomer, gave the ion at $m / z 483.0950$ through the HRF mechanism, whereas the ion at $m / z$ 305.0665, corresponding to the monomeric unit, was from QM (quinone methide) fission with interflavanic bond cleavage. The RDA derived B-ring loss confirmed the presence of a pyrogallol moiety [36].

Compounds 15 and 17 were tentatively (epi)gallocatechin gallate isomers. The TOF-MS/MS spectra of both the compounds showed the loss of A-ring of the flavanolic unit (-126 Da), whereas the loss of the [gallic acid- $\mathrm{H}_{2} \mathrm{O}$ ] moiety provided the fragment ion at $m / z 305.0673(78)$. The presence of gallic acid was further confirmed by the fragment ion at $m / z$ 169.0146(51), which was for compound 15 the base peak of the TOF-MS/MS spectrum (Figure S4A; [35,37]). A similar fragmentation pathway characterized compound 26, which was tentatively identified as epicatechin gallate, whose TOF-MS/MS spectrum also showed the gallate ion as base peak (Figure S4B). Compound 35, identified as epiafzelechin gallate, preferentially lost the dehydrated gallic acid residue, providing the ion at $m / z 273.0777$ as the base peak (Figure S5). The relative abundance of the fragment ion at $m / z 183.0308(1)$ and the presence of the radical ion at $m / z 168.0070$ suggested for compounds 23 and 34 the occurrence of a methyl gallate bonded through ester bond to the alcoholic function in C-3 of epigallocatechin and epicatechin, respectively. All identified catechins are common to the extracts prepared with the exception of the metabolite 15, which was detected only in the TeaGNP and TeaCEC samples. Epicatechin (14) is present in a slightly higher percentage in the Japanese Sencha green tea (TeaSNC), Bancha Japanese green tea (TeaBNC), and TeaMTC extracts, while metabolite 17 is more contained in TeaCEC (1.34-, 1.22-, 1.22-, 1.37- and 1.38-fold than Chinese green tea blend in filter (TeaTWF), TeaGNP, TeaSNC, TeaBNC, and TeaMTC, respectively). Epicatechin gallate is equally abundant in TeaCEC, where it was 1.6-fold more than that in TeaMTC.

Metabolites 12 and 13 were identified in chlorogenic acid (5-O-caffeoyl quinic acid, 5-CQA) and coumaroyl quinic acid [38,39]. If compound $\mathbf{1 2}$ is present only in traces in TeaCEC, the metabolite 13, most contained in TeaGNP, was completely absent in the extract obtained from the supplement. Indeed, 5-CQA was identified as the main chlorogenic acid in tea, and its content was found to vary depending on the type of tea (white, green, black tea, and mate) and the processing technology [40].

The other identified metabolites belong to the class of glycosylated flavones and flavonols (Table 3). These substances, which resulted in trace amounts or complete absence in TeaCEC, mainly showed quercetin and kaempferol as aglycone moiety in mono-, di- and tri-saccharidic compounds. In particular, the metabolites 16 and 19 are the di-C-glycoside of the apigenin flavone (Figure S6). The TOF-MS/MS spectrum of the ion $[\mathrm{M}-\mathrm{H}]^{-}$at $m / z 593.1555$ for compound 16 underwent the loss of 90 and 120 Da according to sugar moiety cross-ring linkage [41], whereas the TOF-MS/MS ion at $m / z 563.1442$ for 19 gave ions at $m / z 503.1225$ [M - H-60] $]^{-}, 473.1113$ [M - H-90] ${ }^{-}$, and 443.1004 $[\mathrm{M}-\mathrm{H}-120]^{-}$. These losses allowed us to hypothesize the presence of saccharide residues linked

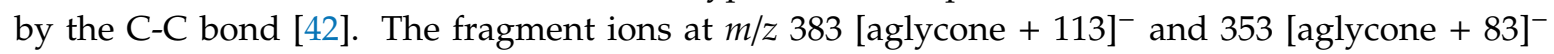
were in accordance with the presence of apigenin as aglycone. Different flavone C-glycosides were previously identified in green tea, and apigenin 6-C-arabinosyl-8-C-glucoside (isoschaftoside) and apigenin 6-C-glucosyl-8-C-arabinoside (schaftoside) were identified among this class of compounds [43]. The relative abundance in TOF-MS/MS spectrum of compound 19 of the ion at $\mathrm{m} / \mathrm{z} 443$ and the absence of 273 ion was in accordance with isoschaftoside occurrence. 
Table 1. TOF-MS and TOF-MS/MS of tentatively identified non-flavonoid compounds in investigated green tea extracts. Compounds are numbered based on their RT in the whole total ion current chromatogram. $\mathrm{RT}=$ Retention Time; RDB $=$ Ring Double Bond equivalent value

\begin{tabular}{|c|c|c|c|c|c|c|c|c|}
\hline 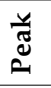 & $\begin{array}{c}\mathrm{RT} \\
(\mathrm{min})\end{array}$ & $\begin{array}{l}\text { Tentative } \\
\text { Assignment }\end{array}$ & Formula & $\begin{array}{l}{[\mathrm{M}-\mathrm{H}]^{-} \text {calc. }} \\
(\mathrm{m} / \mathrm{z})\end{array}$ & $\begin{array}{c}{[\mathrm{M}-\mathrm{H}]^{-}} \\
\text {Found }(\mathrm{m} / \mathbf{z})\end{array}$ & $\begin{array}{r}\text { Error } \\
(\mathrm{ppm})\end{array}$ & RDB & $\begin{array}{l}\text { Fragment Ions } \\
\text { MS/MS (m/z) }\end{array}$ \\
\hline 1 & 0.304 & pentosylhexoside & $\mathrm{C}_{11} \mathrm{H}_{20} \mathrm{O}_{10}$ & 311.0984 & 311.0992 & 2.7 & 2 & $311.1004 ; 179.0560 ; 161.0453 ; 87.0086$ \\
\hline 2 & 0.310 & theanin & $\mathrm{C}_{7} \mathrm{H}_{14} \mathrm{~N}_{2} \mathrm{O}_{3}$ & 173.0932 & 173.0932 & 0.2 & 2 & 155.0836; 93.0350; 84.0459; 82.0313 \\
\hline 3 & 0.316 & quinic acid & $\mathrm{C}_{7} \mathrm{H}_{12} \mathrm{O}_{6}$ & 191.0561 & 191.0564 & 1.5 & 2 & $\begin{array}{c}\text { 191.0564; 173.0451; 171.0292; 127.0399; 111.0451; 93.0347; } \\
\text { 87.0087; 85.0298; 83.0500; 81.0344 }\end{array}$ \\
\hline 4 & 0.319 & trihexoside & $\mathrm{C}_{18} \mathrm{H}_{32} \mathrm{O}_{16}$ & 503.1618 & $\begin{array}{r}503.1637 \\
{[\mathrm{M}+\mathrm{Cl}]^{-}}\end{array}$ & 3.7 & 3 & $\begin{array}{l}\text { 503.1654; 341.1097; 323.0983; 281.0891; 251.0789; 221.0667; } \\
\quad \text { 179.0558; 161.0451; } 119.0349 ; 113.0246 ; 89.0242\end{array}$ \\
\hline 5 & 0.324 & dihexoside & $\mathrm{C}_{12} \mathrm{H}_{22} \mathrm{O}_{11}$ & 341.1089 & 341.1102 & 3.7 & 2 & $\begin{array}{l}\text { 341.1095; 179.0556; 161.0452; 149.0446; 143.0345; 131.0352; } \\
\text { 119.0348; 113.0244; 101.0245; 95.0140; 89.0247 }\end{array}$ \\
\hline 6 & 0.873 & gallic acid & $\mathrm{C}_{7} \mathrm{H}_{6} \mathrm{O}_{5}$ & 169.0142 & 169.0148 & 3.3 & 5 & 125.0242 \\
\hline 7 & 1.049 & theogallin & $\mathrm{C}_{14} \mathrm{H}_{16} \mathrm{O}_{10}$ & 343.0671 & 343.0671 & 0.1 & 7 & $191.0561 ; 127.0397 ; 85.0290$ \\
\hline 12 & 2.515 & $\begin{array}{l}\text { 5-O-caffeoyl } \\
\text { quinic acid }\end{array}$ & $\mathrm{C}_{16} \mathrm{H}_{18} \mathrm{O}_{9}$ & 353.0877 & 353.0878 & -0.3 & 8.0 & $191.0558 ; 179.0345 ; 173.0459 ; 135.0448 ; 85.0291$ \\
\hline 13 & 2.734 & $\begin{array}{l}\text { coumaroyl quinic } \\
\text { acid }\end{array}$ & $\mathrm{C}_{16} \mathrm{H}_{18} \mathrm{O}_{8}$ & 337.0931 & 337.0929 & 0.6 & 8.0 & $191.0561 ; 173.0452 ; 163.0401 ; 119.0497 ; 93.0344$ \\
\hline
\end{tabular}


Table 2. TOF-MS and TOF-MS/MS of tentatively identified flavanols in investigated green tea extracts. Compounds are numbered based on their RT in the whole total ion current chromatogram. RT = Retention Time; RDB = Ring Double Bond equivalent value.

\begin{tabular}{|c|c|c|c|c|c|c|c|c|}
\hline ฮँ & $\begin{array}{c}\mathrm{RT} \\
(\mathrm{min})\end{array}$ & $\begin{array}{c}\text { Tentative } \\
\text { Assignment }\end{array}$ & Formula & $\begin{array}{l}{\left[\mathrm{M}-\mathrm{H}^{-} \text {calc. }\right.} \\
\quad(\mathrm{m} / \mathrm{z})\end{array}$ & $\begin{array}{c}{[\mathrm{M}-\mathrm{H}]^{-}} \\
\text {Found }(\mathrm{m} / \mathrm{z})\end{array}$ & $\begin{array}{r}\text { Error } \\
(\mathrm{ppm})\end{array}$ & RDB & $\begin{array}{l}\text { Fragment Ions } \\
\text { MS/MS (m/z) }\end{array}$ \\
\hline 8 & 1.400 & gallocatechin & $\mathrm{C}_{15} \mathrm{H}_{14} \mathrm{O}_{7}$ & 305.0667 & 305.0671 & 1.4 & 9 & $\begin{array}{c}305.0678 ; 221.0463 ; 219.0672 ; 177.0561 ; 167.0356 ; 139.0407 ; \\
137.0251 ; 125.0253 ; 109.0300\end{array}$ \\
\hline 9 & 1.771 & prodelphinidin B & $\mathrm{C}_{30} \mathrm{H}_{26} \mathrm{O}_{14}$ & 609.1250 & 609.1265 & 2.5 & 18 & $\begin{array}{c}\text { 609.1294; 483.0950; 441.0835; 423.0733; 355.0804; 305.0665; } \\
\text { 255.0305; 221.0454; 179.0340; 177.0180; } 125.0236\end{array}$ \\
\hline 10 & 2.094 & epigallocatechin & $\mathrm{C}_{15} \mathrm{H}_{14} \mathrm{O}_{7}$ & 305.0667 & 305.0671 & 1.4 & 9 & $\begin{array}{c}305.0671 ; 219.0667 ; 167.0353 ; 139.0403 ; 137.0248 ; 125.0248 ; \\
109.0299\end{array}$ \\
\hline 11 & 2.320 & catechin & $\mathrm{C}_{15} \mathrm{H}_{14} \mathrm{O}_{6}$ & 289.0718 & 289.0722 & 1.5 & 9 & $\begin{array}{l}289.0727 ; 245.0825 ; 221.0821 ; 205.0514 ; 203.0719 ; 187.0402 ; \\
179.0353 ; 151.0407 ; 137.0251 ; 125.0248 ; 123.0457 ; 109.0300\end{array}$ \\
\hline 14 & 2.904 & epicatechin & $\mathrm{C}_{15} \mathrm{H}_{14} \mathrm{O}_{6}$ & 289.0718 & 289.0722 & 1.5 & 9 & $\begin{array}{l}289.0733 ; 245.0830 ; 221.0831 ; 205.0515 ; 203.0723 ; 187.0409 ; \\
179.0358 ; 151.0405 ; 137.0250 ; 125.0247 ; 123.0456 ; 109.0299\end{array}$ \\
\hline 15 & 3.074 & $\begin{array}{l}\text { (epi)gallocatechin } \\
\text { gallate } 1\end{array}$ & $\mathrm{C}_{22} \mathrm{H}_{18} \mathrm{O}_{11}$ & 457.0776 & 457.0786 & 2.1 & 14 & $\begin{array}{c}331.0463 ; 305.0673 ; 219.0668 ; 193.0148 ; 169.0146 ; 161.0245 ; \\
137.0245 ; 125.0246\end{array}$ \\
\hline 17 & 3.357 & $\begin{array}{l}\text { (epi)gallocatechin } \\
\text { gallate } 2\end{array}$ & $\mathrm{C}_{22} \mathrm{H}_{18} \mathrm{O}_{11}$ & 457.0776 & 457.0788 & 2.5 & 14 & $\begin{array}{c}331.0476 ; 305.0678 ; 219.0668 ; 193.0148 ; 169.0151 ; 161.0249 ; \\
137.0246 ; 125.0250\end{array}$ \\
\hline 23 & 3.606 & $\begin{array}{l}\text { epigallocatechin } \\
\text { methylgallate }\end{array}$ & $\mathrm{C}_{23} \mathrm{H}_{20} \mathrm{O}_{11}$ & 471.0943 & 471.0933 & 2.2 & 14 & $\begin{array}{c}305.0678 ; 219.0667 ; 183.0308 ; 179.0355 ; 168.0070 ; 161.0251 ; \\
125.0249\end{array}$ \\
\hline 26 & 3.691 & epicatechin gallate & $\mathrm{C}_{22} \mathrm{H}_{18} \mathrm{O}_{10}$ & 441.0827 & 441.0836 & 2.0 & 14 & $\begin{array}{c}\text { 441.0841; 331.0461; } 303.0508 ; 289.0715 ; 245.0811 ; 203.0707 ; \\
\text { 193.0137; 169.0138; } 151.0393 ; 137.0236 ; 125.0241 ; 124.0162 ; \\
109.0291\end{array}$ \\
\hline 34 & 4.008 & $\begin{array}{l}\text { epicatechin } \\
\text { methylgallate }\end{array}$ & $\mathrm{C}_{23} \mathrm{H}_{20} \mathrm{O}_{10}$ & 455.0995 & 455.0984 & 2.5 & 14 & $289.0724 ; 245.0819 ; 183.0301 ; 168.0070 ; 125.0242$ \\
\hline 35 & 4.018 & $\begin{array}{l}\text { epiafzelechin } \\
\text { gallate }\end{array}$ & $\mathrm{C}_{22} \mathrm{H}_{18} \mathrm{O}_{9}$ & 425.0881 & 425.0883 & 0.7 & 14 & $\begin{array}{c}\text { 425.0910; 287.0574; } 273.0777 ; 255.0666 ; 243.0690 ; 211.0760 ; \\
169.0144 ; 125.0246 ; 97.0297\end{array}$ \\
\hline
\end{tabular}


Table 3. TOF-MS and TOF-MS/MS of tentatively identified flavonol glycosides in investigated green tea extracts. Compounds are numbered based on their RT in the whole total ion current chromatogram. $\mathrm{RT}=$ Retention Time; $\mathrm{RDB}=$ Ring Double Bond equivalent value.

\begin{tabular}{|c|c|c|c|c|c|c|c|c|}
\hline हूँ & $\begin{array}{c}t_{R} \\
(\min )\end{array}$ & Tentative Assignment & Formula & $\begin{array}{l}{[\mathrm{M}-\mathrm{H}]-\text { calc. }} \\
(\mathrm{m} / \mathbf{z})\end{array}$ & $\begin{array}{l}{[\mathrm{M}-\mathrm{H}]^{-}} \\
\text {Found }(\mathrm{m} / \mathrm{z})\end{array}$ & $\begin{array}{l}\text { Error } \\
(\mathrm{ppm})\end{array}$ & RDB & $\begin{array}{l}\text { Fragment Ions } \\
\text { MS/MS (m/z) }\end{array}$ \\
\hline 16 & 3.191 & $\begin{array}{l}\text { apigenin 6-C-hexosyl- } \\
\text { 8-C-hexoside }\end{array}$ & $\mathrm{C}_{27} \mathrm{H}_{30} \mathrm{O}_{15}$ & 593.1512 & 593.1520 & 1.4 & 13 & $\begin{array}{c}\text { 593.1555; 575.1398; 545.1276; 503.1207; } 473.1109 ; 395.0780 ; 383.0779 ; \\
\text { 353.0672; 325.0715; 297.0771; 221.0452; } 191.0357\end{array}$ \\
\hline 18 & 3.377 & myricetin galloyl hexoside & $\mathrm{C}_{28} \mathrm{H}_{24} \mathrm{O}_{17}$ & 631.0941 & 631.0950 & 1.5 & 17 & 631.0979; 479.0853; 317.0294; 316.0225; 287.0192; 271.0239; 178.9978; 169.0139 \\
\hline 20 & 3.497 & myricetin deoxyhexosyl hexoside & $\mathrm{C}_{27} \mathrm{H}_{30} \mathrm{O}_{17}$ & 625.1410 & 625.1422 & 1.9 & 13 & $\begin{array}{c}\text { 625.1468; 463.0914; 317.0314; 316.0237; 300.0286; 271.0263; 214.0264; } \\
\text { 178.9990; } 151.0039\end{array}$ \\
\hline 21 & 3.503 & myricetin hexoside & $\mathrm{C}_{21} \mathrm{H}_{20} \mathrm{O}_{13}$ & 479.0831 & 479.0847 & 3.3 & 12 & $479.0867 ; 317.0309 ; 316.0238 ; 287.0201 ; 271.0256 ; 214.0274 ; 178.9993$ \\
\hline 24 & 3.633 & quercetin trihexoside & $\mathrm{C}_{33} \mathrm{H}_{40} \mathrm{O}_{21}$ & 771.1989 & 771.2004 & 1.9 & 14 & $\begin{array}{c}\text { 771.2031; 609.1496; 343.0461; 301.0356; 300.0278; 271.0247; 255.0298; } \\
\text { 178.9990; } 151.0036\end{array}$ \\
\hline 25 & 3.681 & quercetin galloyl hexoside & $\mathrm{C}_{28} \mathrm{H}_{24} \mathrm{O}_{16}$ & 615.0992 & 615.0998 & 1.0 & 17 & $615.1061 ; 463.0920 ; 313.0598 ; 301.0369 ; 300.0287 ; 271.0258 ; 255.0315 ; 179.0003$ \\
\hline 27 & 3.703 & rutin & $\mathrm{C}_{27} \mathrm{H}_{30} \mathrm{O}_{16}$ & 609.1461 & 609.1469 & 1.3 & 13 & $\begin{array}{c}\text { 609.1504; 343.0473; 301.0364; 300.0286; } 271.0256 ; 255.0304 ; 178.9988 ; \\
169.0146 ; 151.0043\end{array}$ \\
\hline 28 & 3.819 & Isoquercetrin & $\mathrm{C}_{21} \mathrm{H}_{20} \mathrm{O}_{12}$ & 463.0888 & 463.0887 & -0.2 & 12 & $\begin{array}{c}463.0919 ; 301.0367 ; 300.0290 ; 299.0200 ; 271.0262 ; 255.0309 ; 243.0310 ; \\
178.9987 ; 151.0038 ; 121.0291\end{array}$ \\
\hline 29 & 3.825 & $\begin{array}{l}\text { kaempferol dihexosyl } \\
\text { deoxyhexoside }\end{array}$ & $\mathrm{C}_{33} \mathrm{H}_{40} \mathrm{O}_{20}$ & 755.2040 & 755.2046 & 0.8 & 14 & $755.2111 ; 285.0411 ; 284.0327 ; 255.0304 ; 229.0507 ; 151.0023$ \\
\hline 30 & 3.842 & quercetin hexoside & $\mathrm{C}_{21} \mathrm{H}_{20} \mathrm{O}_{12}$ & 463.0882 & 463.0887 & 1.1 & 12 & $463.0928 ; 301.0373 ; 300.0293 ; 271.0262 ; 255.0310 ; 243.0305 ; 151.0040$ \\
\hline 32 & 3.959 & $\begin{array}{l}\text { kaempferol hexosyl } \\
\text { deoxyhexoside }\end{array}$ & $\mathrm{C}_{27} \mathrm{H}_{30} \mathrm{O}_{15}$ & 593.1531 & 593.1512 & 3.2 & 13 & $593.1553 ; 285.0407 ; 284.0329 ; 255.0301 ; 227.0349$ \\
\hline 33 & 4.007 & $\begin{array}{l}\text { methylkaempferol } \\
\text { deoxyhexoside }\end{array}$ & $\mathrm{C}_{21} \mathrm{H}_{20} \mathrm{O}_{11}$ & 447.0933 & 447.0942 & 2.0 & 12 & $300.0291 ; 285.0409 ; 284.0333 ; 255.0303 ; 227.0353$ \\
\hline 36 & 4.386 & camelliquercitoside B & $\mathrm{C}_{42} \mathrm{H}_{46} \mathrm{O}_{23}$ & 917.2357 & 917.2361 & 0.4 & 20 & $917.2433 ; 771.2075 ; 753.1915 ; 301.0355 ; 300.0277 ; 299.0198 ; 271.0246 ; 178.9977$ \\
\hline 37 & 4.419 & camelliquercitoside A & $\mathrm{C}_{47} \mathrm{H}_{54} \mathrm{O}_{27}$ & 1049.2780 & 1049.2789 & 0.9 & 21 & $1049.2868 ; 903.2491 ; 885.2401 ; 301.0361 ; 300.0285 ; 299.0200$ \\
\hline 38 & 4.420 & quercetin coumaroyl hexoside & $\mathrm{C}_{30} \mathrm{H}_{26} \mathrm{O}_{14}$ & 609.1250 & 609.1258 & 1.3 & 18 & $\begin{array}{c}\text { 609.1308; 463.0900; 301.0358; 300.0278; 271.0240; 255.0319; 227.0371; } \\
179.0004 ; 151.0030\end{array}$ \\
\hline 39 & 4.447 & camelliquercitoside C & $\mathrm{C}_{41} \mathrm{H}_{44} \mathrm{O}_{22}$ & 887.2251 & 887.2263 & 1.3 & 20 & $\begin{array}{l}\text { 887.2336; 741.1947; 723.1846; 573.1334; 301.0361; 300.0282; 299.0196; } \\
\text { 271.0260; 255.0289; 178.9988; 151.0039 }\end{array}$ \\
\hline 40 & 4.550 & camelliquercitoside D & $\mathrm{C}_{36} \mathrm{H}_{36} \mathrm{O}_{18}$ & 755.1829 & 755.1838 & 1.2 & 19 & $\begin{array}{c}\text { 755.1883; 609.1495; 591.1391; 301.0359; 300.0276; 299.0193; 271.0242; } \\
\text { 255.0292; 178.9979; } 151.0033\end{array}$ \\
\hline 41 & 4.553 & camellikaempferoside C & $\mathrm{C}_{42} \mathrm{H}_{46} \mathrm{O}_{22}$ & 901.2408 & 901.2419 & 1.2 & 20 & $\begin{array}{c}\text { 901.2487; 755.2105; 737.2002; 615.1976; } 285.0412 ; 284.0333 ; 283.0251 ; \\
\text { 229.0525; } 187.0387 ; 145.0297\end{array}$ \\
\hline 42 & 4.654 & kaempferol coumaroylhexoside & $\mathrm{C}_{30} \mathrm{H}_{26} \mathrm{O}_{13}$ & 593.1301 & 593.1325 & 4.1 & 18 & 593.1322; 447.0948; 285.0394; 284.0322; 255.0289; 227.0334; 145.0279 \\
\hline
\end{tabular}


The metabolites 21, 28, 30, 31, and 33 have been identified as monoglycosyl derivatives of flavonols myricetin (21, [44]), quercetin (28 and 30), kaempferol (31), and methylkaempferol (33). The TOF-MS/MS spectrum of compound 21 is in Figure 3C, whereas the TOF-MS/MS spectra of the two quercetin hexosides are reported in Figure 4 (panels D and E). In all cases, the loss of the dehydrated hexose residue led to the formation of radical aglycone as the base peak, leaving the localization of the saccharide unit in C-3 of the aglycone to be hypothesized. The presence of hexose derivatives of the three flavonols-myricetin, quercetin, and kaempferol-has long been reported in tea leaves. Takino et al. [45] reported that hexose sugars could consist of glucose and its epimers galactose and mannose. Compound 18 with the $[\mathrm{M}-\mathrm{H}]^{-}$ion at $m / z 631.0950$ was tentatively identified as myricetin galloylhexoside (Figure 3A). To strengthen this hypothesis, the loss of 152 Da provided the fragment ion at $m / z$ 479.0853, which gave rise to the aglycone anion at $m / z$ 317.0294, which is attributable to myricetin, and its aglycone radical anion at $m / z$ 316.0225. The abundance of this latter favored the hexose moiety to be localized at the C-3 carbon, as for compound 21 (Figure 3C). The acyl derivatives of flavonol monoglycoside were further compounds 25, 38, and 42. Compound 25 showed neutral losses similar to that of compound 18, according to quercetin galloylhexoside (Figure 4B). Compounds 38 and 42 were tentatively identified, based on their TOF-MS/MS spectra, in a quercetin coumaroylhexoside and kaempferol coumaroylhexoside, respectively. The $[\mathrm{M}-\mathrm{H}]^{-}$ion of compound 38 at $\mathrm{m} / \mathrm{z} 609.1258$ (Figure 5C) was in accordance with the molecular formula $\mathrm{C}_{30} \mathrm{H}_{26} \mathrm{O}_{14}$, whereas the deprotonated molecular ion of compound 42 suggested the molecular formula $\mathrm{C}_{30} \mathrm{H}_{26} \mathrm{O}_{13}$. The loss of dehydrated coumaric acid was ascertained through TOF-MS/MS experiments.

Compounds 27 and 32 were putatively identified as quercetin-3-O-rutinoside (Figure 4C) and kaempferol-7-O-rutinoside, respectively. Compounds $\mathbf{2 0}$ and $\mathbf{2 2}$ were tentatively identified as myricetin diglycosides, differing in the glyconic moiety, which appeared to be constituted by a rutinosyl residue in compound 20 (Figure 3B). Two hexose residues characterized compound 22, which, based on the high abundance of the ion at $m / z$ 479.0857, derived from the loss of the first hexosyl moiety, were each hypothesized to be located on C-3 or C-7 carbons (Figure 3D).

The remaining compounds present a greater complexity of the non-aglyconic portion. In particular, compound 24, showing the deprotonated molecular ion at $\mathrm{m} / \mathrm{z} 771.2004$, in accordance with the molecular formula $\mathrm{C}_{33} \mathrm{H}_{40} \mathrm{O}_{21}$, provided, in the TOF-MS ${ }^{2}$ experiment, the [aglycone- $\left.\mathrm{H}\right]^{-}$ion and the most abundant radical aglycone, consisting with the loss of a triglycoside residue linked to the -OH enol group in $\mathrm{C}-3$ of quercetin, whose identity was established on the basis of the detection of ions $\left[\mathrm{M}-\mathrm{H}-\mathrm{CH}_{2} \mathrm{O}\right]^{-}$and $\left[\mathrm{M}-\mathrm{H}-\mathrm{CH}_{2} \mathrm{O}_{2}\right]^{-}$at $m / z 271.0247$ and 255.0298 , respectively (Figure $4 \mathrm{~A}$ ). The TOF-MS/MS spectrum of compound 29 was in agreement with the presence of a kaempferol trisaccharide. Accordingly, previous studies suggested the occurrence of a kaempferol triglycoside in which the saccharide moiety consisted of (3"-O-galactosyl)rutinose [46]. The content of these molecules was more abundant in the TeaMTC sample. Recent studies have also found triglycosides of myricetin and have defined their disappearance as a consequence of the fermentation and oxidation processes [46]. Recently, the obtainment of a flavonol glycosides-rich extract named FLG was optimized from an aqueous green tea extract after tannase treatment [47]. Apigenin-C-mono- and diglycosides, as well as some different mono- and diglycosides of flavonols myricetin, quercetin, and kaempferol were detected.

The metabolites $36,37,39$, and 40 were further identified as acylglycosides of the flavonol quercetin (Figure 5). In particular, the $[\mathrm{M}-\mathrm{H}]^{-}$ion of metabolite 36 at $\mathrm{m} / \mathrm{z} 917.2361$ dissociated by supplying the fragment ions at $m / z 771.2075$ and 753.1915 , according to the neutral loss of a residue of coumaric acid- $\mathrm{H}_{2} \mathrm{O}$ and coumarate (Figure 5A). This compound was recognized as camelliquercitoside B. The [M $-\mathrm{H}]^{-}$ion at $m / z 1049.2789$ for compound 37 was in line with camelliquercitoside A occurrence, whereas compound 39 could be camelliquercitoside C. In all the TOF-MS/MS spectra of these compounds, the $[\mathrm{M}-\mathrm{H}]^{-}$ion dissociated providing both the $\left[\mathrm{M}-\mathrm{H} \text {-coumaric acid- } \mathrm{H}_{2} \mathrm{O}\right]^{-}$and $[\mathrm{M}-\mathrm{H}$-coumaric acid $]^{-}$ions, whose ratio appeared to vary depending on saccharide moiety. This finding was in line with a common localization of the hydroxycinnamoyl moiety on the sugar component directly linked 
to the aglycone. Instead, compound $\mathbf{4 0}$ was tentatively identified as camelliquercitoside D (Figure 5E). The $[\mathrm{M}-\mathrm{H}]^{-}$ion underwent a loss of coumaroyl moiety to achieve the ion at $m / z 609.1495$, which was likely rutin. Finally, compound $\mathbf{4 1}$ was putatively a coumaroyl derivative of compound $\mathbf{2 9}$ (Figure 6). In fact, the detected [M - H-coumaric acid- $\left.\mathrm{H}_{2} \mathrm{O}\right]^{-}$ion at $\mathrm{m} / \mathrm{z} 755.2105$ could lose 470 Da to provide the deprotonated kampferol aglycone. It was putatively identified as camellikaempferoside $\mathrm{C}$, which is an acylated flavonol tetraglycoside that was recently isolated from Lu'an GuaPian green tea [48]. The identification of acylated flavonol di- and tryglycosides in different tea types is quite recent. In particular, flavonol mono- and diglycosides were reported as functional food ingredients that are able to regulate postprandial hyperglycemia through the inhibition of $\alpha$-glucosidase/ $\alpha$-amylase [49].

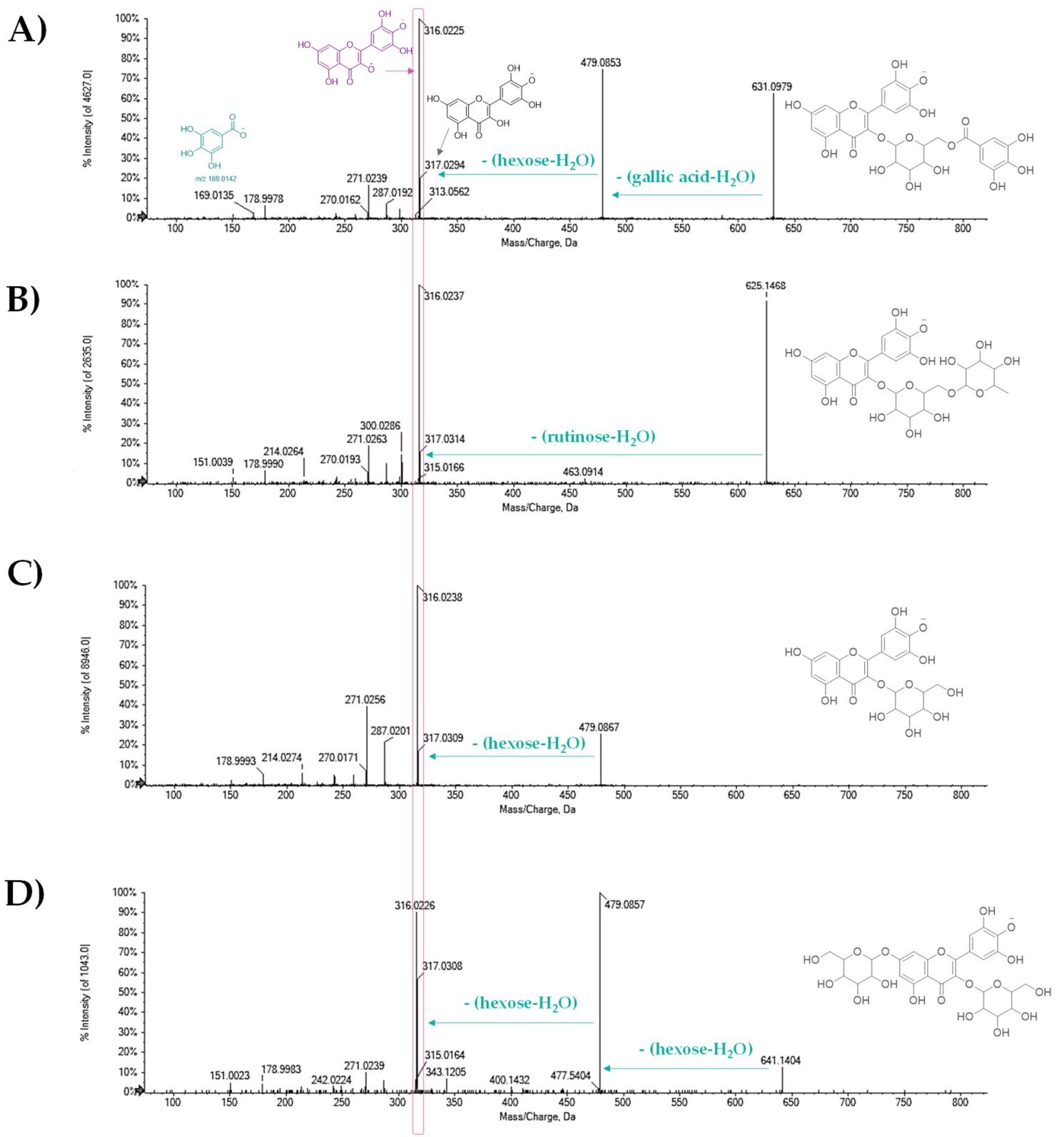

Figure 3. TOF-MS/MS spectra of [M - H] $]^{-}$ion of myricetin glycosides (A) 18, (B) 20, (C) 21, and (D) 22. The structure of the deprotonated molecular ion is reported on the left, whereas the main neutral losses are evidenced by green arrays. 


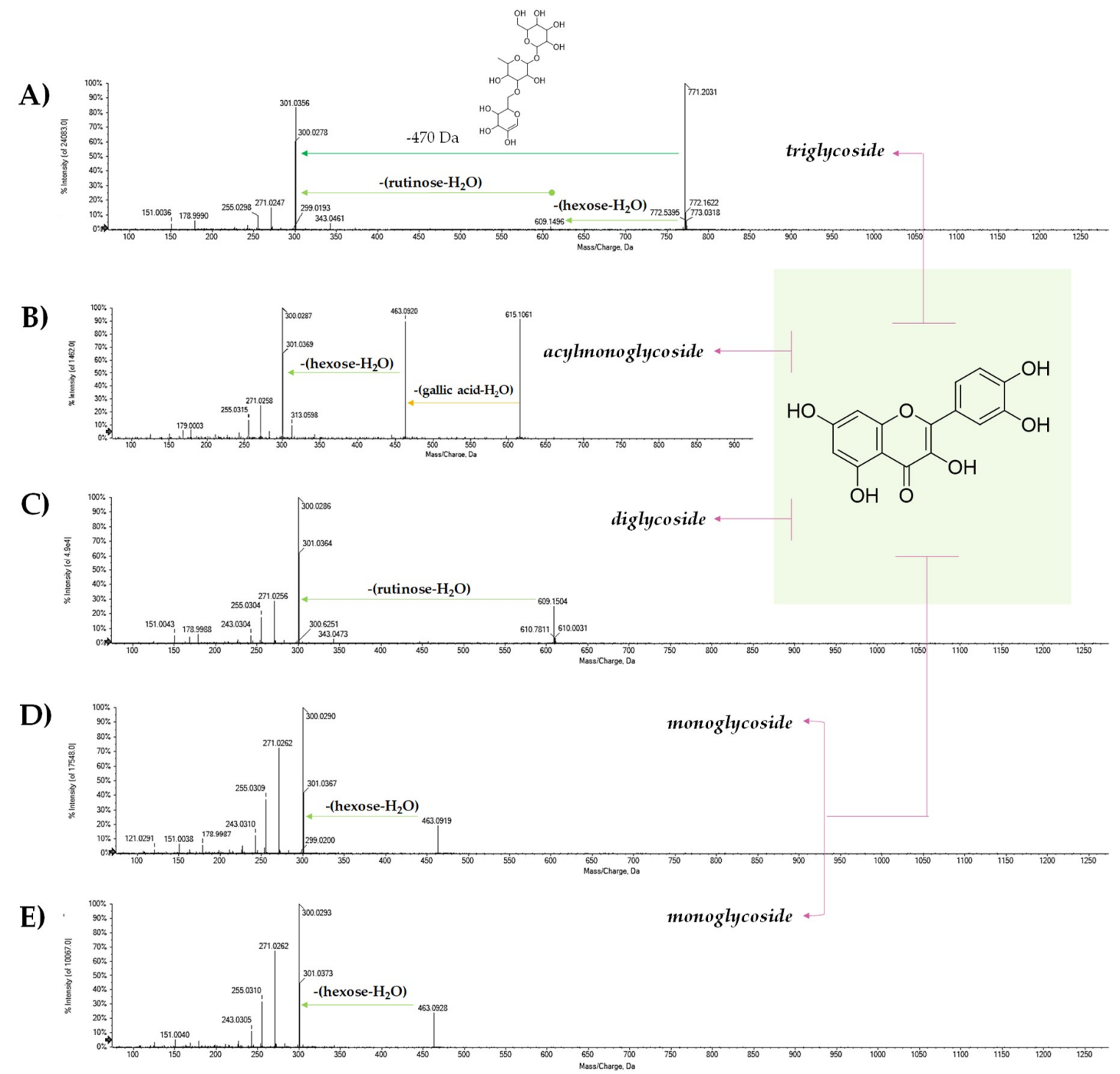

Figure 4. TOF-MS/MS spectra of $[\mathrm{M}-\mathrm{H}]^{-}$ion of quercetin glycosides (A) 24, (B) 25, (C) 27, (D) 28, and (E) 30 . 
A)

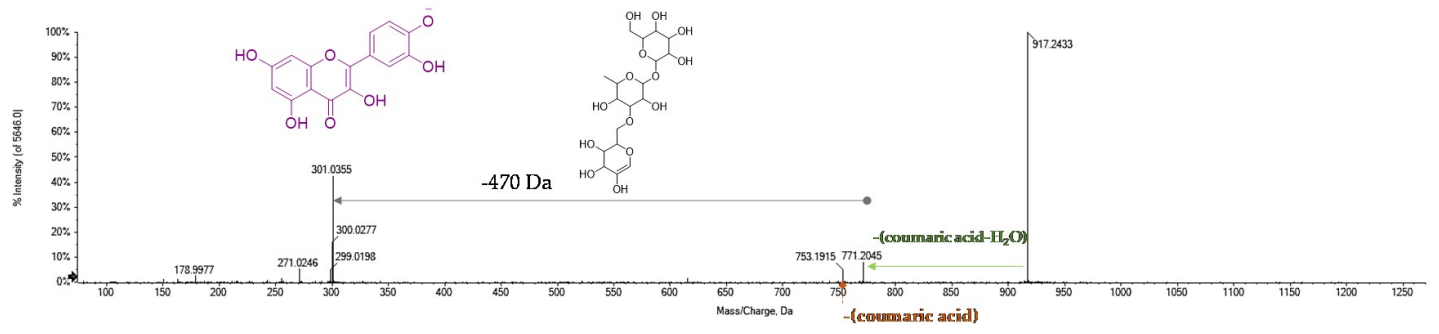

B)

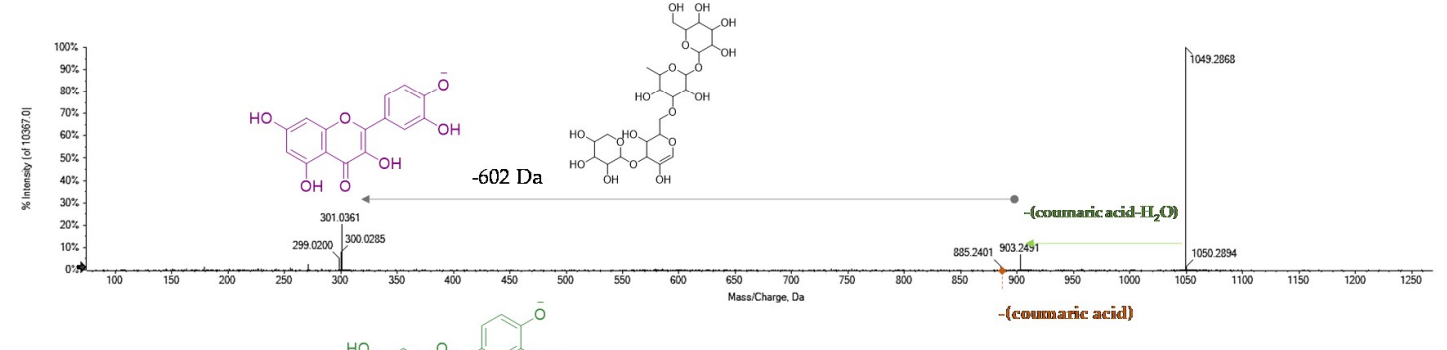

C)

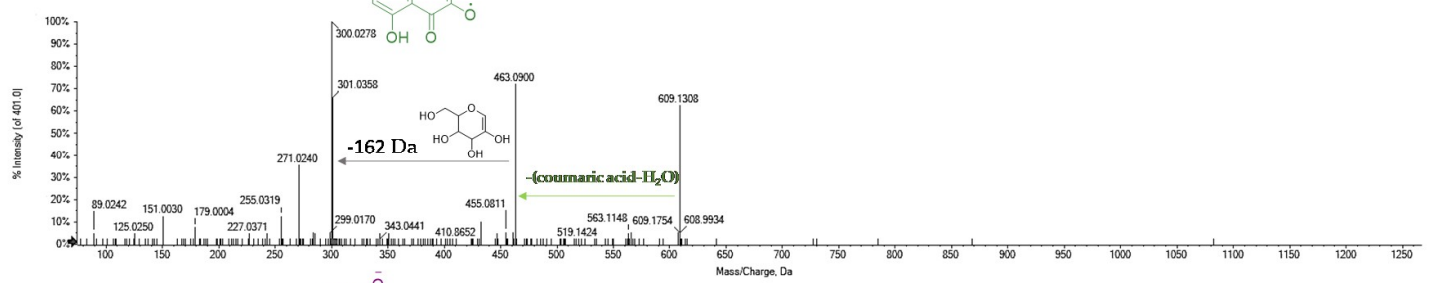

D)

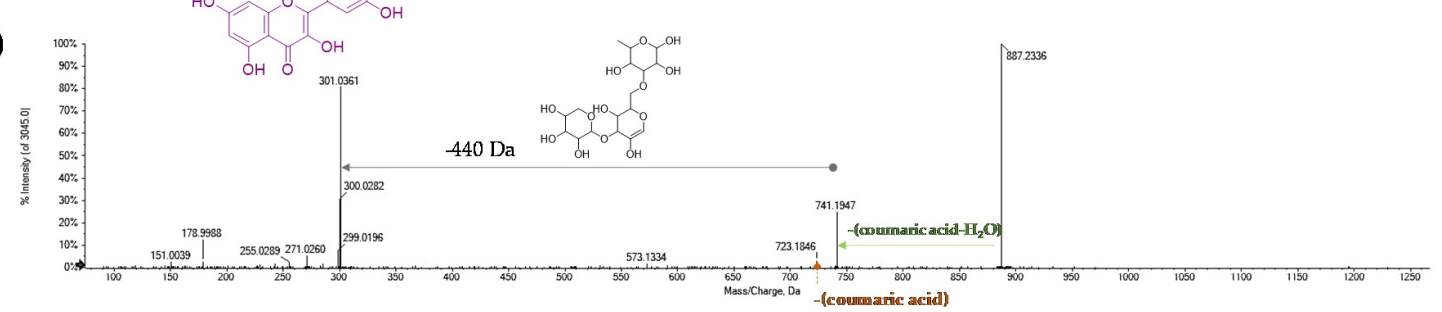

E)

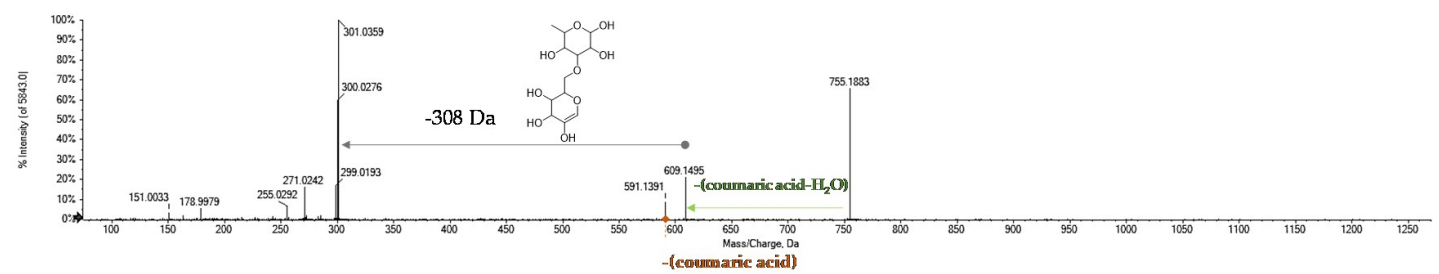

Figure 5. TOF-MS/MS spectra of $[\mathrm{M}-\mathrm{H}]^{-}$ion of some of tentatively identified quercetin coumaroyl glycosides (A) 36, (B) 37, (C) 38, (D) 39, and (E) 40.

Kaempferol coumaroyl glycosides, such as camellikaempferoside B, were reported as a constituent of Fuzhuan brick tea, which is a kind of uniquely microbial fermented tea in China [50]. This compound was found to possess the properties of both kaempferol and p-coumaric acid and significantly inhibit $A \beta$ production by decreasing $\beta$-secretase activity [51]. Their biosynthesis is mediated by an acyltransferase, namely $\mathrm{CsHCT}$, that can catalyze the transfer of an acyl group to the donor substrate [52]. Acyl flavonoid glycosides were identified as active ingredients with good inhibitory abilities on $\alpha$-glucosidase and HMG-CoA reductase, and the hypoglycemic and hypolipidemic effects of Fuzhuan brick tea were attributable to their presence [53]. 

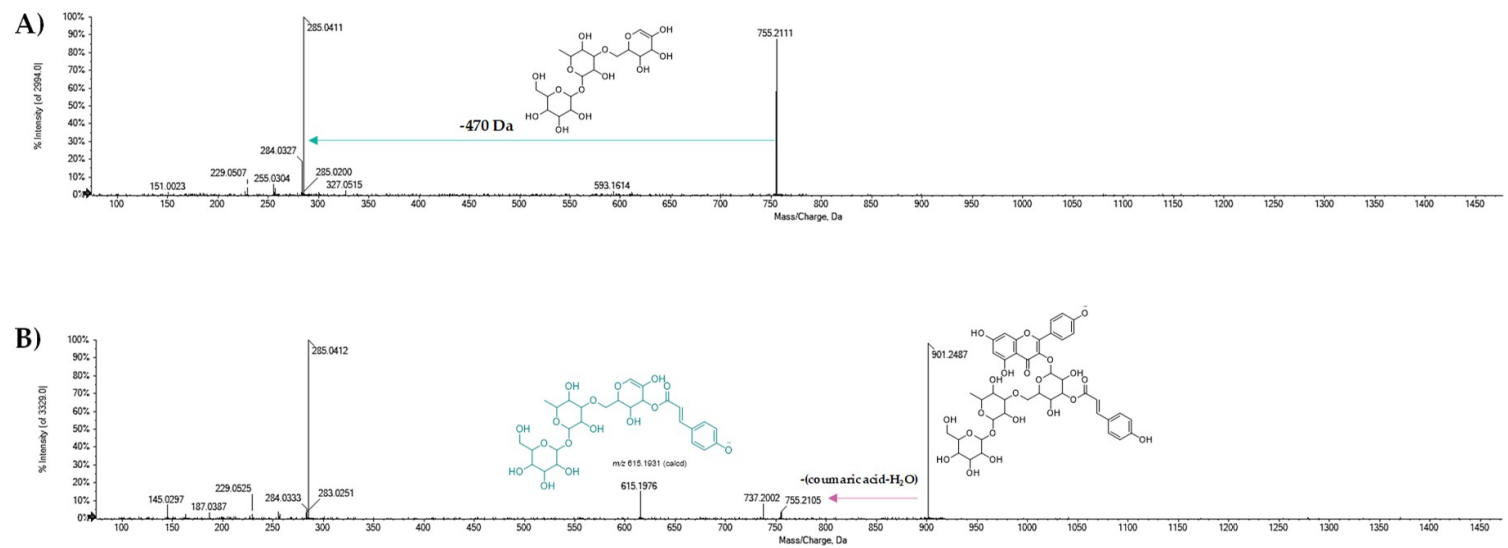

Figure 6. TOF-MS/MS spectra of $[\mathrm{M}-\mathrm{H}]^{-}$ion of some of kaempferol triglycosides (A) 29 and (B) 41. In the B panel, the presence of a coumaroyltryglycosyl moiety was highlighted by detecting the ion at $m / z 615.1976$.

\subsection{Estimation of Radical Scavenging, Antioxidant, and Antilipoperoxidant Activity}

Alcoholic green tea extracts were also compared for their antiradical and antilipoperoxidant capabilities, although aware that, on the basis of the chemical composition, the TeaCEC extract, presenting the non-flavonoid component only in traces and completely lacking in the flavonol glycosides, could exert a more marked efficacy in the employed test tubes. In fact, tea catechins were broadly investigated for their antioxidant properties, and several studies defined the structural variants responsible for this activity [54]. Catechins reactivity in cell-free systems is attributable to the catechol/pyrogalloyl function on the B-ring, and their antiradical efficacy is greatly increased by esterification with a residue of gallic acid [55,56]. Indeed, large discrepancies are found in the literature among different green teas, suggesting that technological processes such as wilting, twisting, or drying, mostly affect the antioxidant properties of the product that reaches the consumer [57]. Thus, the co-presence in the obtained extracts of flavonolic glycosylated molecules and saccharides favored the comparative execution of four widely employed tests in the chemical antioxidant scenario. The anti-free radical tests $\mathrm{DPPH}^{\bullet}$ and $\mathrm{ABTS}^{+\bullet}$ were based on a mixed mechanism of action, given by the ability of a molecule to inactivate radical species with mechanisms of transfer of a hydrogen atom (HAT) or a single electron (SET). Data acquired, which clearly showed the dose-dependent radical scavenging action of the tested samples, are graphed in Figure 7 (panels A and B). The results from the most responsive ABTS assay evidenced that TeaCEC was able to almost completely reduce the probe species at a $3.125 \mu \mathrm{g} / \mathrm{mL}$ dose level; meanwhile, the TeaBNC and TeaSNC samples exerted a comparable reducing effect. Based on DPPH and $\mathrm{ABTS}_{\mathrm{ID}} \mathrm{D}_{50}$ values, it could be stated that the antiradical activity is negatively affected by the content of saccharide and quinic acid in the extracts. 
A)

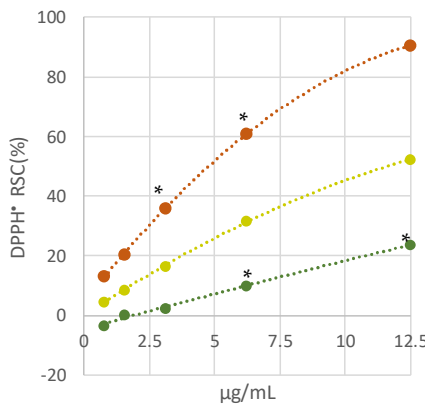

$\bullet$ TeaCEC $\bullet$ TeaTWF $\bullet$ TeaSNC

B)

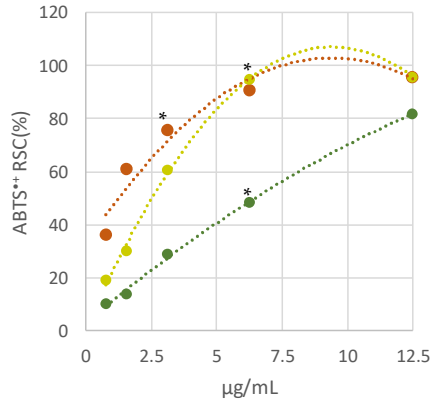

- TeaCEC • TeaTWF - TeaSNC

C)

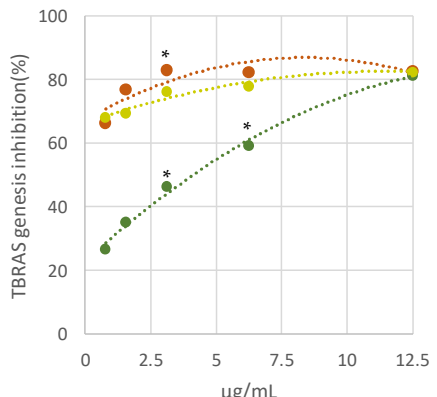

$\bullet$ TeaCEC • TeaTWF • TeaSNC

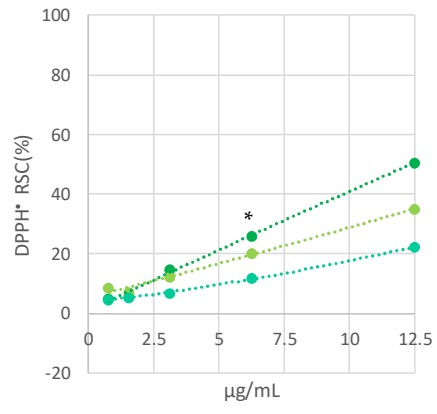

$\bullet$ TeaBNC $\bullet$ TeaMTC $\bullet$ TeaGNP

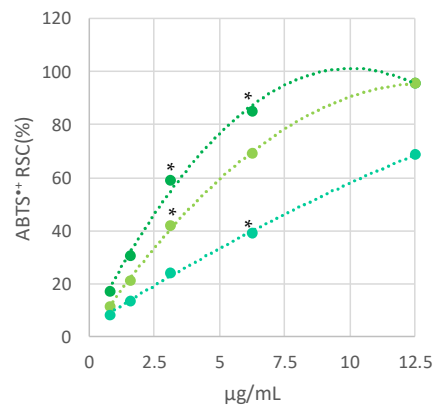

- TeaBNC - TeaMTC - TeaGNP

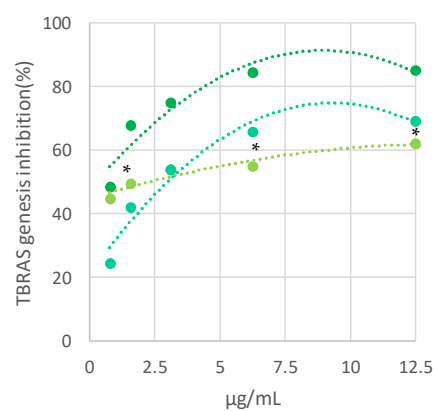

- TeaBNC - TeaMTC • TeaGNP

D)

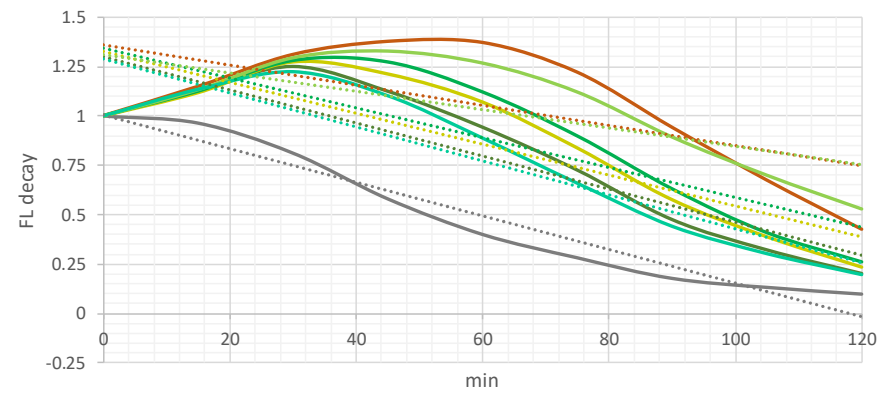

二

Figure 7. Radical Scavenging Capacity (RSC, \%) of green tea extracts vs. 2,2'-diphenyl-1-picrylhydrazyl) (DPPH) radical (A) and 2,2'-azino-bis(3-ethylbenzothiazoline-6-sulfonic acid (ABTS) radical cation (B). Inhibition of thiobarbituric acid reactive substances (TBARS) species formation (\%; C). Values are the mean \pm SD of two independent experiments performed in triplicate. ${ }^{*} p<0.05$ vs. blank. (D) Fluorescein fluorescence decay induced by the 2,2'-azobis-(2-amidinopropane)-dihydrochloride (AAPH) radical generator. The decay is strongly delayed by the co-presence of the extracts already at $0.78 \mu \mathrm{g} / \mathrm{mL}$ dose. 
The TBARS assay further demonstrated that green tea extracts effectively counteracted a UV-induced lipoxidation of hemp oil, which is highly rich in Polyunsaturated Fatty Acids (PUFA), and therefore highly unstable (Figure 7C). Data obtained were in line with the results by Lotito and Fraga [58], who, analyzing the effectiveness of pure catechins, found that they were effective antioxidants in human blood plasma, being able to delay lipoperoxidation and depletion of fat-soluble endogenous antioxidants such as $\beta$-carotene and $\alpha$-tocopherol. Finally, an ORAC test also confirmed that all the extracts were able to prevent the formation of peroxyl radicals causing an increase in the fluorescein fluorescence. Curves obtained by the co-exposure of the radical generator, fluorescein, and extracts prepared at the lowest tested dose are in Figure 7D. TeaGNP and TeaTWF again appeared to be the less active extracts. Indeed, while TGP showed an antiradical trend closer to the other extracts, the TWF behavior was totally different. In spite of what was observed for the other two tests aimed at estimating the anti-free radical activity, TeaSNC was slightly less active than TeaBNC, and both are less effective than TeaMTC and TeaCEC.

The relative abundance of each class of compounds reasonably affects the antioxidant response. Catechin content in the partially purified TeaCEC extract reached the $89.9 \%$, and it was 1.60-, 1.66-, 1.57-, 1.51-, 1.86-fold more abundant than in TeaTWF, TeaMTC, TeaSNC, TeaBNC, and TeaGNP, whereas its sugar amount was 14.1- and 13.0-fold lower than that in the less active TeaTWF and TeaGNP extracts. The higher relative content in catechins and chlorogenic acids of TeaBNC reasonably favored, in spite of its important sugar rate (11.0\%), a good antiradical and antilipoperoxidant activity. It is noteworthy to be highlighted that TeaSNC, which showed a catechin content similar to that of TeaBNC, accounted for almost half of the sugars. The Relative Antioxidant Capacity Index (RACI), whose calculation represents the average of the standard scores obtained from the raw data for the various methods, comprehensively emphasized that, taking into account TeaCEC exception, TeaSNC was more active than the others (Table 4 ).

Table 4. Antioxidant activity of green tea extracts expressed as $\operatorname{ID}_{50}(\mu \mathrm{g} / \mathrm{mL})$ and TEAC (Trolox ${ }^{\circledR}$ Equivalent Antioxidant Capacity values, $\mathrm{ID}_{50}$ Trolox ${ }^{\circledR} / \mathrm{ID}_{50}$ sample $)$ vs. DPPH and $\mathrm{ABTS}^{+}$radicals. $\mathrm{ID}_{50}$ and Trolox ${ }^{\circledR}$ Equivalent Antioxidant Capacity (TEAC) values from TBARS inhibition assay, as well as oxygen radical absorbance capacity (ORAC) values (Trolox ${ }^{\circledR} \mathrm{Eq} \mu \mathrm{M}$ ) are also reported. Relative Antioxidant Capacity Index (RACI) values, which were calculated taking into account the average of the standard scores obtained from the $\mathrm{ID}_{50}$ raw data for the various methods and ORAC values, are also given in the last column. TeaCEC: green tea catechins-enriched supplement, TeaTWF: Chinese green tea blend in filter, TeaSNC: Japanese Sencha green tea, TeaBNC: Japanese Bancha green tea, TeaMTC: Matcha green tea, TeaGNP: gunpowder green tea.

\begin{tabular}{|c|c|c|c|c|c|}
\hline & $\begin{array}{l}\text { ID }_{50} \text { DPPH } \\
(\mu \mathrm{g} / \mathrm{mL}) \text { TEAC }\end{array}$ & $\begin{array}{l}\text { ID }_{50} \text { ABTS } \\
(\mu \mathrm{g} / \mathrm{mL}) \text { TEAC }\end{array}$ & $\begin{array}{l}\text { ID }_{50} \text { TBARS } \\
(\mu \mathrm{g} / \mathrm{mL}) \text { TEAC }\end{array}$ & $\begin{array}{c}\text { ORAC (Trolox }{ }^{\circledR} \\
\text { Eq } \mu \mathrm{M})\end{array}$ & RACI \\
\hline TeaCEC & $\begin{array}{c}3.83 \pm 0.25 \\
3.91\end{array}$ & $\begin{array}{c}1.13 \pm 0.12 \\
4.42\end{array}$ & $\begin{array}{c}0.17 \pm 0.01 \\
38.2\end{array}$ & $3.71 \pm 0.16$ & 9.96 \\
\hline TeaTWF & $\begin{array}{c}66.52 \pm 0.25 \\
0.22\end{array}$ & $\begin{array}{c}5.28 \pm 0.72 \\
0.95\end{array}$ & $\begin{array}{l}3.14 \pm 0.61 \\
2.04\end{array}$ & $2.72 \pm 0.16$ & -0.16 \\
\hline TeaSNC & $\begin{array}{c}15.37 \pm 0.51 \\
0.97\end{array}$ & $\begin{array}{c}2.26 \pm 0.32 \\
2.20\end{array}$ & $\begin{array}{c}0.28 \pm 0.01 \\
22.8\end{array}$ & $3.57 \pm 0.63$ & 5.62 \\
\hline TeaBNC & $\begin{array}{l}19.71 \pm 0.38 \\
0.76\end{array}$ & $\begin{array}{c}2.44 \pm 0.44 \\
2.04\end{array}$ & $\begin{array}{c}0.55 \pm 0.04 \\
11.5\end{array}$ & $2.53 \pm 0.79$ & 2.36 \\
\hline TeaMTC & $\begin{array}{c}31.15 \pm 0.25 \\
0.48\end{array}$ & $\begin{array}{c}3.35 \pm 0.53 \\
1.49\end{array}$ & $\begin{array}{c}1.79 \pm 0.12 \\
3.57\end{array}$ & $2.72 \pm 0.32$ & 0.32 \\
\hline TeaGNP & $\begin{array}{c}83.11 \pm 0.15 \\
0.18\end{array}$ & $\begin{array}{c}7.80 \pm 1.39 \\
0.64\end{array}$ & $\begin{array}{c}2.90 \pm 0.56 \\
2.20\end{array}$ & $2.02 \pm 0.21$ & -0.44 \\
\hline
\end{tabular}

To get an overview, hierarchical clustering analysis was also applied using antiradical and antilipoperoxidant data, as well as the relative content of each class of compounds in investigated extracts (Figure 8). As mentioned above, differences in composition and therefore in bioactivity may depend on the different geographical location of the plantations and on the different methods of processing and refinement of the leaf. There is no information on conservation methods before 
purchase, but unpredictable exposure to heat or incorrect storage can lead to the destruction of some polyphenolic and non-polyphenolic compounds, or to epimerization reactions [13].

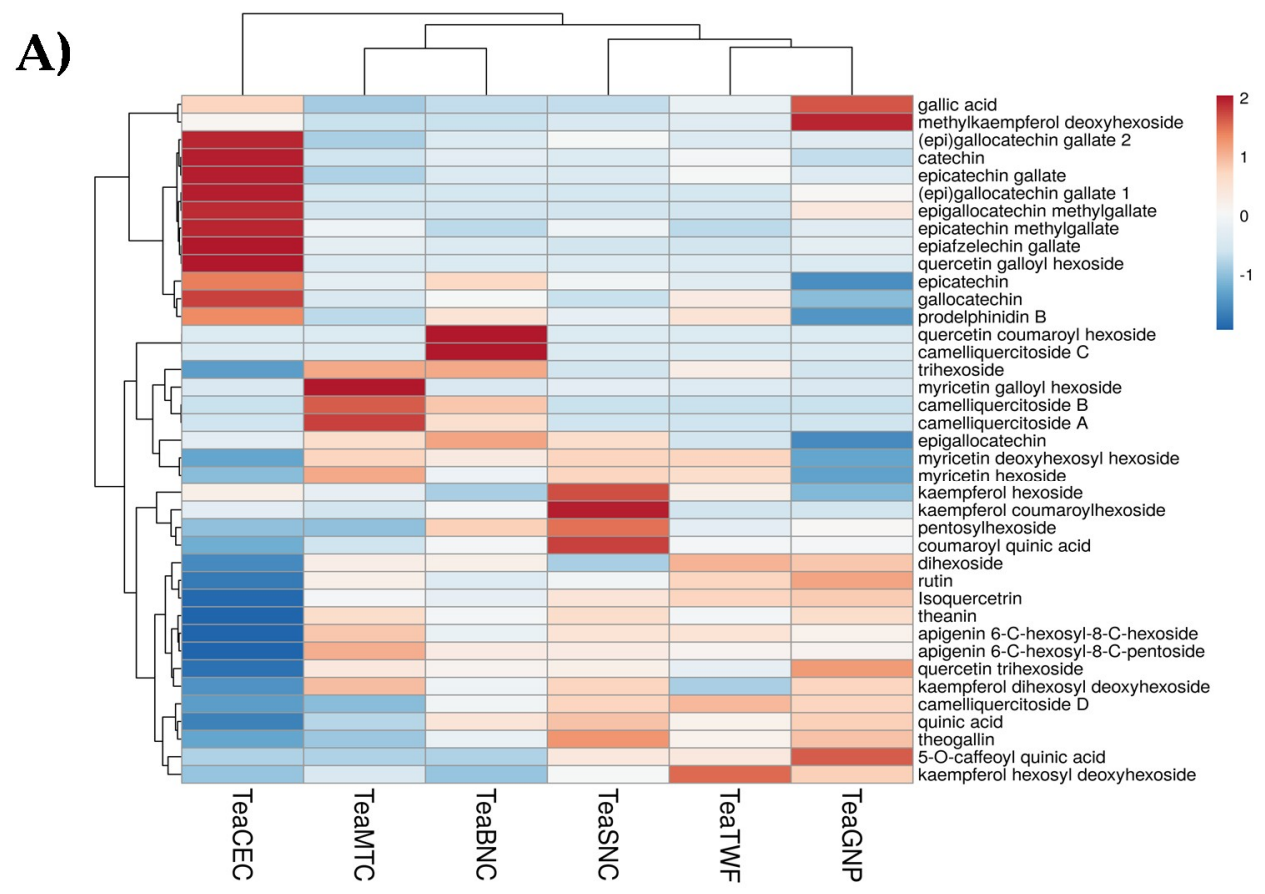

B)

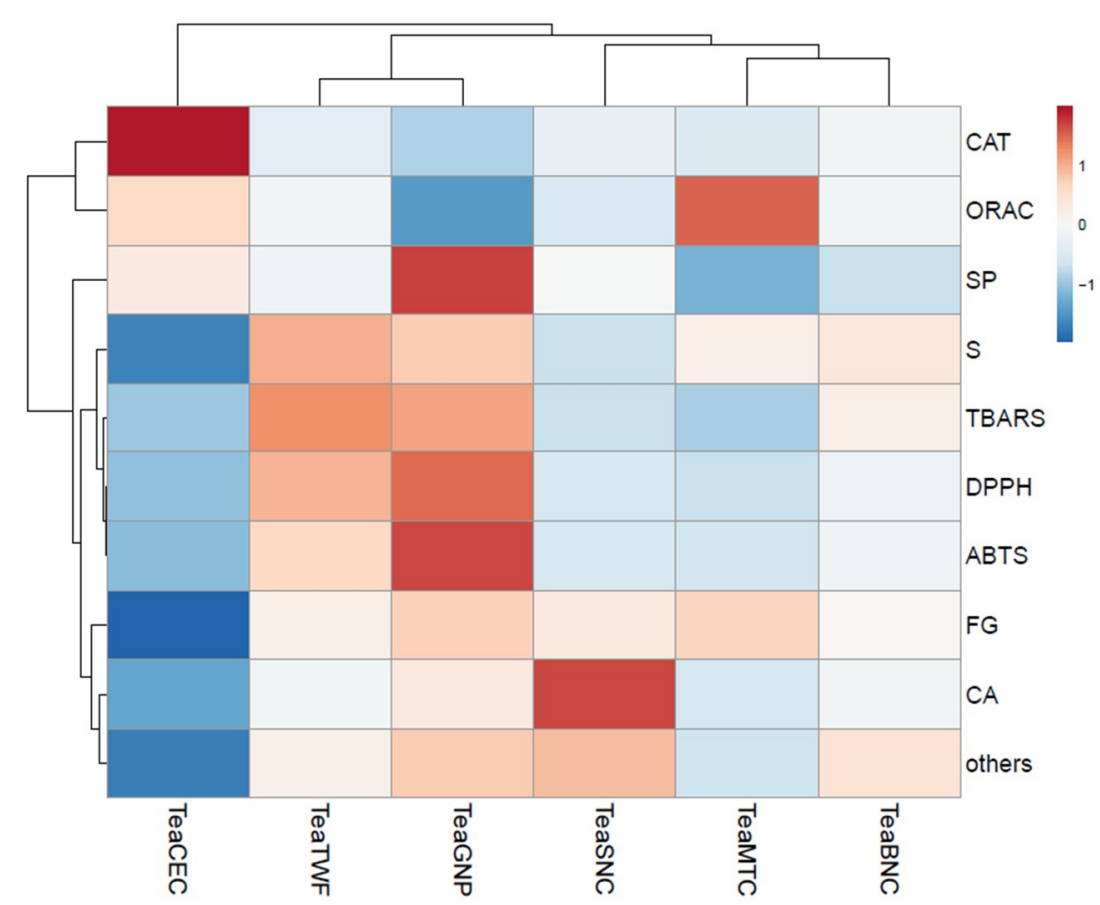

Figure 8. Heatmap (A) of compounds tentatively identified in alcoholic tea extracts; (B) relative content of each class of compounds $(\mathrm{S}=$ Sugars, $\mathrm{SP}=$ Simple Phenols; $\mathrm{CA}=$ Chlorogenic Acids; CAT = Catechins; FG = Flavonol Glycosides; "others" is for theanin and quinic acid). Annotations on top of the heatmap show clustering of the tea samples. In the ClustVis hierarchical clustering tool, both rows and columns are clustered using correlation distance and average linkage [59]. 


\section{Materials and Methods}

\subsection{Green Tea Samples and Preparation of their Alcoholic Extracts}

Commercial green tea leaves from different geographical areas and processing were the focus of the present investigation. All the matrices were bought from a supermarket or herbalist's shop. In particular, Chinese green tea blend in filter (throughout the text referred to as TeaTWF), Chinese gunpowder green tea (TeaGNP), Japanese Sencha green tea (TeaSNC), Japanese Bancha green tea (TeaBNC) and Japanese Matcha green tea (TeaMTC) were analyzed together along with the green tea-based supplement (TeaCEC), which was reported to be titrated to $80 \%$ of total catechins, of which $50 \%$ were EGCG.

Tea samples (10 g each) underwent ultrasound accelerated maceration (Branson UltrasonicsTM Bransonic $^{\mathrm{TM}}$ M3800-E, Danbury, CT, USA), using ethanol as the extracting solvent in a ratio tea leaf:solvent equal to 1:5. In order to obtain the complete recovery of the tea leaf metabolic content, five sonication cycles were performed (30 min each) on all the tea samples, except for MTC, which was subjected to four sonication cycles, and CEC, whose catechins' recovery was after one only cycle. At the end of each sonication cycle, samples were centrifuged at $3500 \times g$ for $10 \mathrm{~min}$ at $4{ }^{\circ} \mathrm{C}$ and then filtered using a paper filter. The extracts obtained were dried under vacuum using a rotary evaporator (Heidolph Hei-VAP Advantage, Schwabach, Germany).

\subsection{UHPLC-ESI-QqTOF-MS and MS/MS Analyses}

The alcoholic tea extracts, reconstituted in methanol LC-MS grade, at a $10 \mathrm{mg} / \mathrm{mL}$ dose level, were analyzed using a Shimadzu NEXERA UHPLC system (Shimadzu, Tokyo, Japan) equipped with a Luna ${ }^{\circledR}$ Omega Polar C18 column $(1.6 \mu \mathrm{m}, 50 \times 2.1 \mathrm{~mm}$ i.d, Phenomenex, Torrance, CA, USA). The separation was achieved using a binary solution $\left.\mathrm{A}) \mathrm{H}_{2} \mathrm{O}(0.1 \% \mathrm{HCOOH}), \mathrm{B}\right) \mathrm{CH}_{3} \mathrm{CN}(0.1 \% \mathrm{HCOOH})$ using a gradient program, which started at $5 \% \mathrm{~B}$, held for $1.5 \mathrm{~min}$, and linearly ramping up to $25 \% \mathrm{~B}$ in $11 \mathrm{~min}$. Then, the initial condition was restored and held for another $0.5 \mathrm{~min}$. The total run time was $13 \mathrm{~min}$, with a flow rate of $0.4 \mathrm{~mL} \mathrm{~min}^{-1}$. The injection volume was $2.0 \mu \mathrm{L}$.

MS analysis was performed using a hybrid Q-TOF MS instrument, the AB SCIEX Triple TOF ${ }^{\circledR}$ 4600 (AB Sciex, Concord, ON, Canada), equipped with a DuoSprayTM ion source (consisting of both electrospray ionization (ESI) and atmospheric pressure chemical ionization (APCI) probes), which was operated in the negative ESI mode. The APCI probe was used for automated mass calibration using the Calibrant Delivery System (CDS, AB Sciex, Concord, ON, Canada). The CDS injects a calibration solution matching the polarity of ionization and calibrates the mass axis of the Triple $\mathrm{TOF}^{\circledR}$ system in all scan functions used (MS and/or MS/MS). The Q-TOF HRMS method, which combines TOF-MS and MS/MS with information-dependent acquisition (IDA) for identifying non-targeted and unexpected compounds, consisted of a full scan TOF survey (dwell time $100 \mathrm{~ms}, 100-1500 \mathrm{Da}$ ) and eight IDA MS/MS scans (dwell time $100 \mathrm{~ms}, 80-1250 \mathrm{Da}$ ). The MS parameters were as follows: curtain gas (CUR) 35 psi, nebulizer gas (GS 1) 60 psi, heated gas (GS 2) 60 psi, ion spray voltage (ISVF) $4.5 \mathrm{kV}$, interface heater temperature (TEM) $600{ }^{\circ} \mathrm{C}$, declustering potential (DP) -75 V. Collision Energy (CE) applied was $-45 \mathrm{~V}$ with a collision energy spread (CES) of $15 \mathrm{~V}$. The instrument was controlled by Analyst ${ }^{\circledR}$ TF 1.7 software (AB Sciex, Concord, ON, Canada, 2016), while data processing was carried out using PeakView ${ }^{\circledR}$ software version 2.2 (AB Sciex, Concord, ON, Canada, 2016).

\subsection{Assessment of the Antioxidant Effectiveness}

Investigated green tea extracts were tested at different dosage levels. Tests were carried out for three replicate measurements each. Recorded activities were compared to a blank treated equally to the samples. The assessment of antioxidant and radical scavenging abilities of investigated tea extracts was carried through four methods. Each extract was estimated at dose levels equal to $(0.78,1.56,3.125$, 6.25 , and $12.5 \mu \mathrm{g} / \mathrm{mL}$ - final concentration levels), and its activity was compared to a blank arranged in parallel to the samples. ABTS [2,2'-azinobis-(3-ethylbenzothiazolin-6-sulfonic acid)] radical cation 
scavenging capacity and 2,2-diphenyl-1-picrylhydrazyl (DPPH) radical scavenging capability were determined as previously reported. TBARS and ORAC methods were also applied. Results are the mean $\pm \mathrm{SD}$ values. $\mathrm{ID}_{50}$ values were calculated.

\subsubsection{Determination of 2,2'-Diphenyl-1-Picrylhydrazyl (DPPH) Radical Scavenging Capacity}

The determination of $\mathrm{DPPH}^{\bullet}$ scavenging capability was estimated as follows: increasing doses of green tea extracts were dissolved in a $\mathrm{DPPH}^{\bullet}$ methanol solution $\left(9.4 \times 10^{-5} \mathrm{M}\right)$ at room temperature. After $15 \mathrm{~min}$ of incubation, the absorption at $517 \mathrm{~nm}$ was measured by a Wallac Victor3 spectrophotometer in reference to a blank. The results are expressed in terms of the percentage reduction of the initial DPPH radical adsorption by green tea test samples. Trolox $(4,8,16,32 \mu \mathrm{M})$ was used as standard [60].

3.3.2. Determination of ABTS [2,2'-Azinobis-(3-Ethylbenzothiazolin-6-Sulfonic Acid)] Radical Cation Scavenging Capacity

The radical cation ABTS was previously generated by the reaction between the diammonium salt of the 2,2'-azinobis-3-etilbenzotiazolin- 6-sulfonic acid (ABTS) $(7.0 \mathrm{mM})$ and potassium persulfate $\left(\mathrm{K}_{2} \mathrm{~S}_{2} \mathrm{O}_{8}\right)(2.45 \mathrm{mM})$, leaving the reaction mixture in the dark for $12 \mathrm{~h}$. Subsequently, the solution containing the radical cation ABTS was diluted in PBS (phosphate-buffered saline) in order to obtain an absorbance of 0.7 at $734 \mathrm{~nm}$. Increasing doses of green tea were dissolved in the ABTS ${ }^{\bullet+}$ solution. After incubation (6 $\mathrm{min}$ ) the absorbance was measured at $734 \mathrm{~nm}$, using a Wallac Victor3 spectrophotometer (PerkinElmer, Waltham, MA, USA) in reference to a blank. Trolox $(4,8,16,32 \mu \mathrm{M})$ was used as standard [61].

\subsubsection{Determination of Thiobarbituric Acid Reactive Substances (TBARS)}

Thiobarbituric acid (TBA) reagent was prepared as follows: for reagent A, TBA (375.0 $\mathrm{mg}$ ) and tannic acid $(30.0 \mathrm{mg})$ were dissolved in hot water $(30.0 \mathrm{~mL})$; for reagent $B$, trichloroacetic acid $(15 \mathrm{~g})$ was dissolved in an aqueous hydrogen chloride solution $(0.30 \mathrm{M}, 70.0 \mathrm{~mL})$. Then, reagent A was mixed with reagent B. Next, hemp oil $(6.0 \mu \mathrm{L})$ was emulsified with Tween-40 $(15.6 \mathrm{mg})$ initially dissolved in Tris- $\mathrm{HCl}$ buffer $(0.2 \mathrm{M}, 2.0 \mathrm{~mL}, \mathrm{pH} 7.4)$. Afterwards, $25 \mu \mathrm{L}$ of each sample $(0.78,3.125,6.25,12.5$, 25.0, $50.0 \mu \mathrm{g} / \mathrm{mL}$; final concentration) and Trolox (2.0, 4.0, 8.0, $16.0 \mu \mathrm{M}$; final concentration) were added to $500 \mu \mathrm{L}$ of reaction mixture. Samples were irradiated with UV light for $60 \mathrm{~min}$ at $254 \mathrm{~nm}$. After addition of the TBA reagent $(1.0 \mathrm{~mL})$, all test tubes were placed in a boiling water bath for $15 \mathrm{~min}$. Then, $300.0 \mu \mathrm{L}$ of $n$-butanol were added and centrifuged for $3 \mathrm{~min}$ at $1500 \mathrm{~g}$. The absorbance of supernatant was measured at $532 \mathrm{~nm}$. The inhibition of lipid peroxidation was recorded as percentage versus a blank containing no test samples. Trolox $(4,8,16,32 \mu \mathrm{M})$ was used as standard [62].

\subsubsection{Determination of Oxygen Radical Absorbance Capacity (ORAC)}

Green tea extracts $(20 \mu \mathrm{L} ; 0.78,1.56$, and $3.125 \mu \mathrm{g} / \mathrm{mL}$, final concentrations) and fluorescein $\left(120.0 \mu \mathrm{L}, 70 \mathrm{nM}\right.$, final concentration) solutions were preincubated for $15 \mathrm{~min}$ at $37^{\circ} \mathrm{C}$ in $75.0 \mathrm{mM}$ phosphate buffer ( $\mathrm{pH}$ 7.4). Then, 2,2' -azobis-(2-amidinopropane)-dihydrochloride (AAPH) solution $(60.0 \mu \mathrm{L}, 12.0 \mathrm{mM}$, final concentration) was rapidly added. In parallel with the samples, a blank $(\mathrm{FL}+\mathrm{AAPH})$ and solutions of the standard antioxidant Trolox $(1-8 \mu \mathrm{M}$, final concentrations) were properly prepared in PBS. The fluorescence $\left(\lambda_{\mathrm{ex}}=485 \mathrm{~nm}, \lambda_{\mathrm{em}}=525 \mathrm{~nm}\right)$ was recorded every $15 \mathrm{~min}$ for 120 min using a Wallac Victor3 (PerkinElmer, Waltham, MA, USA). Antioxidant curves (fluorescence versus time) were first normalized to the curve of the blank by multiplying original data by the factor fluorescence blank, $t=0$ / fluorescence sample, $t=0$. From the normalized curves, the area under the fluorescence decay curve (AUC) was calculated as follows: $\mathrm{AUC}=\sum \mathrm{fi} / \mathrm{f0}$, where $\mathrm{f} 0$ was the initial fluorescence reading at $0 \mathrm{~min}$ and fi was the fluorescence reading at time $i$. Linear regression equations between net $\mathrm{AUC}\left(\mathrm{AUC} \mathrm{C}_{\text {antioxidant }}-\mathrm{AUC}_{\text {blank }}\right)$ and antioxidant concentration were calculated for all the samples [60]. 


\subsection{Statistical Analysis}

Antiradical and antilipoperoxidant assays were performed three times with replicate samples, except where otherwise indicated. Data are expressed as mean $\pm \mathrm{SD}$ (standard deviation). The means were compared using analysis of variance (ANOVA) plus Bonferroni's t-test. A $p$-value of $<0.05$ was considered to indicate a statistically significant result. The Relative Antioxidant Capacity Index (RACI) was calculated according to the method of Sun and Tanumihardjo [63]. The standard score was calculated as follows: $(x-\mu) / \sigma$ where $x$ is the raw data, $\mu$ is the mean, and $\sigma$ is the standard deviation. Standard scores have a mean of 0 and standard deviation equal to 1 . ClustVis, freely available at http://biit.cs.ut.ee/clustvis/, was used for data pre-processing and heatmap plotting.

\section{Conclusions}

Green tea, highly consumed worldwide, and with broadly proven properties, beyond catechins, is a reservoir of flavonol glycosides and their acylated derivatives. The content of these substances, together with that of chlorogenic acids, simple phenols, and saccharides is highly variable in marketed products, based on tea type and quality leaf. UAM extraction, together with HR MS/MS analyses, proved to maximize their disclosure, also when an extracting solvent that is not properly suitable for this class of compounds was used. The relative abundance of each class of compounds reasonably correlated to the antioxidant response. The antiradical activity was found to be negatively affected by saccharides and quinic acid amount in the extracts, in spite of the presence of catechins and/or other polyphenols. This finding was clearly appreciated when alcoholic extracts from TeaSNC and TeaBNC were compared. In fact, the first one proved to be more active than the second, although showing a similar catechin content but accounting for almost half of the sugars. The preventive role of flavonoid compounds makes green tea products, if properly standardized, a favorable tool to counteract oxidative stress conditions onset, in association with a healthy and correct lifestyle. Furthermore, the data acquired addresses the research for new extraction strategies that better lead to a full understanding of acylated flavonol glycosides content in other tea types. Moreover, the good solubility in water of flavonol glycosides and their acylated derivatives lays the foundation for identifying and quantifying them when herbal tea preparation is through call brewing.

Supplementary Materials: The following are available online. Figure S1: Total Ion Chromatograms (TICs) of alcoholic extracts • TeaTWF; • TeaGNP; • TeaSNC; TeaBNC; • TeaMTC; • TeaCEC. Figure S2: TOF-MS/MS spectra of deprotonated compound $\mathbf{1}(\mathrm{A})$, and $[\mathrm{M}+\mathrm{Cl}]^{-}$adducts of compounds $4(\mathrm{~B})$ and $5(\mathrm{C})$. In the D panel, the formation of the ion at $m / z 221$ is proposed based on raffinose trihexose; $m / z$ values, below each structure, are the calculated ones. Figure S3: TOF-MS/MS spectra of $[\mathrm{M}-\mathrm{H}]^{-}$ion of compound (A) 8, and (C) 10. In panel B, the fragmentation pattern that leads to the HRF-mediated formation of the fragment ion at $\mathrm{m} / z 125.0244$ (calcd) is exemplified (in blue), whereas the RDA reaction gives the fragment ions at $m / z 137.0244$ e 167.0350 (calcd). This latter fragment ion is diagnostic for gallocatechins and all the derivatives showing a pyrogallol B-ring. Figure S4: TOF-MS/MS spectra of $[\mathrm{M}-\mathrm{H}]^{-}$ion of compound (A) 15, and (B) 26. The structure of the main fragment ions is highlighted; $m / z$ values, below each structure are the calculated ones. Figure S5: TOF-MS/MS spectrum of [M $\mathrm{H}]^{-}$ion of compound 35. The neutral loss of dehydrated gallic acid residue is highlighted; $m / z$ values, below each structure, are the calculated ones. Figure S6: TOF-MS/MS spectra of $[\mathrm{M}-\mathrm{H}]^{-}$ion of compound (A) 16 and (B) 19. The structures assigned to the compounds are reported in the grey panel.

Author Contributions: Conceptualization, L.C. and S.P. (Severina Pacifico); methodology, L.C., S.P. (Simona Piccolella), and S.P. (Severina Pacifico); validation, L.C., and M.F.; formal analysis, L.C., G.C., and M.F.; investigation, L.C., G.C., and M.F.; data curation, L.C., S.P. (Simona Piccolella), and S.P. (Severina Pacifico); writing-original draft preparation, L.C. and S.P. (Severina Pacifico); writing-review and editing, S.P. (Simona Piccolella) and S.P. (Severina Pacifico); visualization, L.C., G.C., and M.F.; supervision, S.P. (Severina Pacifico). All authors have read and agreed to the published version of the manuscript.

Funding: This research received no external funding.

Conflicts of Interest: The authors declare no conflict of interest. 


\section{References}

1. Sigley, G. Tea and China's rise: Tea, nationalism and culture in the 21st century. Int. Commun. Chin. Cult. 2015, 2, 319-341. [CrossRef]

2. FAO. Global tea consumption and production driven by robust demand in China and India. 2018. Available online: http://www.fao.org/news/story/en/item/1136255/icode/ (accessed on 22 March 2020).

3. Chacko, S.M.; Thambi, P.T.; Kuttan, R.; Nishigaki, I. Beneficial effects of green tea: A literature review. Chin. Med. 2010, 5, 13. [CrossRef] [PubMed]

4. Reygaert, W.C. Green Tea Catechins: Their Use in Treating and Preventing Infectious Diseases. Biomed. Res. Int. 2018, 2018, 9105261. [CrossRef] [PubMed]

5. Li, Q.; Zhao, H.; Zhao, M.; Zhang, Z.; Li, Y. Chronic green tea catechins administration prevents oxidative stress-related brain aging in C57BL/6J mice. Brain Res. 2010, 1353, 28-35. [CrossRef]

6. Roychoudhury, S.; Agarwal, A.; Virk, G.; Cho, C.L. Potential role of green tea catechins in the management of oxidative stress-associated infertility. Reprod. Biomed. Online 2017, 34, 487-498. [CrossRef]

7. Bernatoniene, J.; Kopustinskiene, D.M. The Role of Catechins in Cellular Responses to Oxidative Stress. Molecules 2018, 23, 965. [CrossRef]

8. Keske, M.A.; Ng, H.L.; Premilovac, D.; Rattigan, S.; Kim, J.A.; Munir, K.; Yang, P.; Quon, M.J. Vascular and metabolic actions of the green tea polyphenol epigallocatechin gallate. Curr. Med. Chem. 2015, 22, 59-69. [CrossRef]

9. Liang, Y.; Ip, M.S.M.; Mak, J.C.W. (-)-Epigallocatechin-3-gallate suppresses cigarette smoke-induced inflammation in human cardiomyocytes via ROS-mediated MAPK and NF-кB pathways. Phytomedicine 2019, 58, 152768. [CrossRef]

10. Fujiki, H.; Watanabe, T.; Sueoka, E.; Rawangkan, A.; Suganuma, M. Cancer Prevention with Green Tea and Its Principal Constituent, EGCG: From Early Investigations to Current Focus on Human Cancer Stem Cells. Mol. Cells 2018, 41, 73-82.

11. Ueda-Wakagi, M.; Nagayasu, H.; Yamashita, Y.; Ashida, A.H. Green Tea Ameliorates Hyperglycemia by Promoting the Translocation of Glucose Transporter 4 in the Skeletal Muscle of Diabetic Rodents. Int. J. Mol. Sci. 2019, 20, 2436. [CrossRef]

12. Li, N.; Taylor, L.S.; Ferruzzi, M.G.; Mauer, L.J. Kinetic study of catechin stability: Effects of pH, concentration, and temperature. J. Agric. Food Chem. 2012, 60, 12531-12539. [CrossRef] [PubMed]

13. Eisenstein, M. Tea's value as a cancer therapy is steeped in uncertainty. Nature 2019, 566, S6-S7. [CrossRef] [PubMed]

14. Ananingsih, V.K.; Sharma, A.; Zhou, W. Green tea catechins during food processing and storage: A review on stability and detection. Food Res. Int. 2013, 50, 469-479. [CrossRef]

15. Jiang, H.; Engelhardt, U.H.; Thräne, C.; Maiwald, B.; Stark, J. Determination of flavonol glycosides in green tea, oolong tea and black tea by UHPLC compared to HPLC. Food Chem. 2015, 183, 30-35. [CrossRef]

16. Wu, Y.; Jiang, X.; Zhang, S.; Dai, X.; Liu, Y.; Tan, H.; Gao, L.; Xia, T. Quantification of flavonol glycosides in Camellia sinensis by MRM mode of UPLC-QQQ-MS/MS. J. Chromatogr. B Analyt. Technol. Biomed. Life Sci. 2016, 1017, 10-17. [CrossRef]

17. Namal Senanayake, S.P.J. Green tea extract: Chemistry, antioxidant properties and food applications-A review. J. Funct. Foods 2013, 5, 1529-1541. [CrossRef]

18. Feng, W.Y. Metabolism of Green Tea Catechins: An Overview. Curr. Drug Metab. 2006, 7, 755-809. [CrossRef]

19. Lambert, J.D.; Sang, S.; Yang, C.S. Possible Controversy over Dietary Polyphenols: Benefits vs Risks. Chem. Res. Toxicol. 2007, 20, 583-585. [CrossRef]

20. Lambert, J.D.; Elias, R.J. The antioxidant and pro-oxidant activities of green tea polyphenols: A role in cancer prevention. Arch. Biochem. Biophys. 2010, 501, 65-72. [CrossRef]

21. EFSA Scientific Cooperation (ESCO) Working Group on Botanicals and Botanical Preparations. ESCO Report: Advice on the EFSA guidance document for the safety assessment of botanicals and botanical preparations intended for use as food supplements, based on real case studies. EFSA Supporting Publications 2009, 7, 280. [CrossRef]

22. Oketch-Rabah, H.A.; Roe, A.L.; Rider, C.V.; Bonkovsky, H.L.; Giancaspro, G.I.; Navarro, V.; Paine, M.F.; Betz, J.M.; Marles, R.J.; Casper, S.; et al. United States Pharmacopeia (USP) comprehensive review of the hepatotoxicity of green tea extracts. Toxicol. Rep. 2020, 7, 386-402. [CrossRef] [PubMed] 
23. Hu, J.; Webster, D.; Cao, J.; Shao, A. The safety of green tea and green tea extract consumption in adults Results of a systematic review. Regul. Toxicol. Pharmacol. 2018, 95, 412-433. [CrossRef] [PubMed]

24. Rusak, G.; Komes, D.; Likić, S.; Horžić, D.; Kovač, M. Phenolic content and antioxidative capacity of green and white tea extracts depending on extraction conditions and the solvent used. Food Chem. 2008, 110, 852-858. [CrossRef] [PubMed]

25. Bakht, M.A.; Geesi, M.H.; Riadi, Y.; Imran, M.; Ali, M.I.; Ahsan, M.J.; Ajmal, N. Ultrasound-assisted extraction of some branded tea: Optimization based on polyphenol content, antioxidant potential and thermodynamic study. Saudi J. Biol. Sci. 2019, 26, 1043-1052. [CrossRef]

26. Both, S.; Chemat, F.; Strube, J. Extraction of polyphenols from black tea-Conventional and ultrasound assisted extraction. Ultrason. Sonochem. 2014, 21, 1030-1034. [CrossRef]

27. Lee, L.S.; Lee, N.; Kim, Y.H.; Lee, C.H.; Hong, S.P.; Jeon, Y.W.; Kim, Y.E. Optimization of ultrasonic extraction of phenolic antioxidants from green tea using response surface methodology. Molecules 2013, 18, 13530-13545. [CrossRef]

28. Vuong, Q.V.; Bowyer, M.C.; Roach, P.D. L-Theanine: Properties, synthesis and isolation from tea. J. Sci. Food Agric. 2011, 91, 1931-1939. [CrossRef]

29. Vuong, Q.V.; Stathopoulos, C.E.; Golding, J.B.; Nguyen, M.H.; Roach, P.D. Optimum conditions for the water extraction of L-theanine from green tea. J. Sep. Sci. 2011, 34, 2468-2474. [CrossRef]

30. Verardo, G.; Duse, I.; Callea, A. Analysis of underivatized oligosaccharides by liquid chromatography/ electrospray ionization tandem mass spectrometry with post-column addition of formic acid. Rapid Commun. Mass Spectrom. 2009, 23, 1607-1618. [CrossRef]

31. Shen, J.; Wang, Y.; Chen, C.; Ding, Z.; Hu, J.; Zheng, C.; Li, Y. Metabolite profiling of tea (Camellia sinensis L.) leaves in winter. Sci. Hortic. 2015, 192, 1-9. [CrossRef]

32. Forrest, G.I.; Bendall, D.S. The distribution of polyphenols in the tea plant (Camellia sinensis L.). Biochem. J. 1969, 113, 741-755. [CrossRef] [PubMed]

33. Kongpichitchoke, T.; Chiu, M.T.; Huang, T.C.; Hsu, J.L. Gallic Acid Content in Taiwanese Teas at Different Degrees of Fermentation and Its Antioxidant Activity by Inhibiting PKC $\delta$ Activation: In Vitro and in Silico Studies. Molecules 2016, 21, 1346. [CrossRef] [PubMed]

34. Dimpfel, W.; Kler, A.; Kriesl, E.; Lehnfeld, R.; Keplinger-Dimpfel, I.K. Source density analysis of the human EEG after ingestion of a drink containing decaffeinated extract of green tea enriched with L-theanine and theogallin. Nutr. Neurosci. 2007, 10, 169-180. [CrossRef] [PubMed]

35. Miketova, P.; Schram, K.H.; Whitney, J.; Li, M.; Huang, R.; Kerns, E.; Valcic, S.; Timmermann, B.N.; Rourick, R.; Klohr, S. Tandem mass spectrometry studies of green tea catechins. Identification of three minor components in the polyphenolic extract of green tea. J. Mass Spectrom. 2000, 35, 860-869. [CrossRef]

36. Piccolella, S.; Crescente, G.; Candela, L.; Pacifico, S. Nutraceutical polyphenols: New analytical challenges and opportunities. J. Pharm. Biomed. Anal. 2019, 175, 112774. [CrossRef]

37. Spáčil, Z.; Nováková, L.; Solich, P. Comparison of positive and negative ion detection of tea catechins using tandem mass spectrometry and ultra-high performance liquid chromatography. Food Chem. 2010, 123, 535-541. [CrossRef]

38. Pacifico, S.; Piccolella, S.; Nocera, P.; Tranquillo, E.; Dal Poggetto, F.; Catauro, M. New insights into phenol and polyphenol composition of Stevia rebaudiana leaves. J. Pharm. Biomed. Anal. 2019, 163, 45-57. [CrossRef]

39. Borrelli, F.; Pagano, E.; Formisano, C.; Piccolella, S.; Fiorentino, A.; Tenore, G.C.; Izzo, A.A.; Rigano, D.; Pacifico, S. Hyssopus officinalis subsp. aristatus: An unexploited wild-growing crop for new disclosed bioactives. Ind. Crop. Prod. 2019, 140, 111594. [CrossRef]

40. Nevena, G.L.; Branislava, R.; Emilia, S.; Dusica, R.; Ivan, N.; Nebojsa, K.; Biljana, B. Determination of 5-caffeoylquinic acid (5-CQA) as one of the major classes of chlorogenic acid in commercial tea and coffee samples. Vojnosanit Pregl. 2015, 72, 1018-1023.

41. Pacifico, S.; Galasso, S.; Piccolella, S.; Kretschmer, N.; Pan, S.-P.; Marciano, S.; Bauer, R.; Monaco, P. Seasonal variation in phenolic composition and antioxidant and anti-inflammatory activities of Calamintha nepeta (L.) Savi. Food Res. Int. 2015, 69, 121-132. [CrossRef]

42. Pacifico, S.; Scognamiglio, M.; D’Abrosca, B.; Piccolella, S.; Tsafantakis, N.; Gallicchio, M.; Ricci, A.; Fiorentino, A. Spectroscopic characterization and antiproliferative activity on HepG2 human hepatoblastoma cells of flavonoid C-glycosides from Petrorhagia velutina. J. Nat. Prod. 2010, 73, 1973-1978. [CrossRef] [PubMed] 
43. Lin, L.Z.; Chen, P.; Harnly, J.M. New phenolic components and chromatographic profiles of green and fermented teas. J. Agric. Food Chem. 2008, 56, 8130-8140. [CrossRef] [PubMed]

44. Piccolella, S.; Bianco, A.; Crescente, G.; Santillo, A.; Chieffi Baccari, G.; Pacifico, S. Recovering Cucurbita pepo cv. 'Lungo Fiorentino' Wastes: UHPLC-HRMS/MS Metabolic Profile, the Basis for Establishing Their Nutraand Cosmeceutical Valorisation. Molecules 2019, 24, 1479. [CrossRef] [PubMed]

45. Takino, Y.; Imagawa, H.; Yoshida, H. Studies on the flavonoids in tea leaves. Agric. Biol. Chem. 1962, 26, 699-704.

46. Lee, M.-K.; Kim, H.-W.; Lee, S.-H.; Kim, J.Y.; Asamenew, G.; Choi, J.; Lee, J.-W.; Jung, H.-A.; Yoo, S.M.; Kim, J.-B. Characterization of catechins, theaflavins, and flavonols by leaf processing step in green and black teas (Camellia sinensis) using UPLC-DAD-QToF/MS. Eur. Food. Res. Technol. 2019, 245, 997-1010. [CrossRef]

47. Rha, C.S.; Jeong, H.W.; Park, S.; Lee, S.; Jung, Y.S.; Kim, D.O. Antioxidative, Anti-Inflammatory, and Anticancer Effects of Purified Flavonol Glycosides and Aglycones in Green Tea. Antioxidants 2019, 8, 278. [CrossRef]

48. Bai, W.X.; Wang, C.; Wang, Y.J.; Zheng, W.J.; Wang, W.; Wan, X.C.; Bao, G.H. Novel Acylated Flavonol Tetraglycoside with Inhibitory Effect on Lipid Accumulation in 3T3-L1 Cells from Lu'an GuaPian Tea and Quantification of Flavonoid Glycosides in Six Major Processing Types of Tea. J. Agric. Food Chem. 2017, 65, 2999-3005. [CrossRef]

49. Hu, F.; Zhou, P.; Wu, H.Y.; Chu, G.X.; Xie, Z.W.; Bao, G.H. Inhibition of $\alpha$-glucosidase and $\alpha$-amylase by flavonoid glycosides from Lu'an GuaPian tea: Molecular docking and interaction mechanism. Food Funct. 2018, 9, 4173-4183. [CrossRef]

50. Tian, Y.Z.; Liu, X.; Liu, W.; Wang, W.Y.; Long, Y.H.; Zhang, L.; Xu, Y.; Bao, G.H.; Wan, X.C.; Ling, T.J. A new anti-proliferative acylated flavonol glycoside from Fuzhuan brick-tea. Nat. Prod. Res. 2016, 30, 2637-2641. [CrossRef]

51. Yang, S.; Liu, W.; Lu, S.; Tian, Y.Z.; Wang, W.Y.; Ling, T.J.; Liu, R.T. A Novel Multifunctional Compound Camellikaempferoside B Decreases A $\beta$ Production, Interferes with A $\beta$ Aggregation, and Prohibits A $\beta$-Mediated Neurotoxicity and Neuroinflammation. ACS Chem. Neurosci. 2016, 7, 505-518. [CrossRef]

52. Sun, C.H.; Yang, C.Y.; Tzen, J.T.C. Molecular Identification and Characterization of Hydroxycinnamoyl Transferase in Tea Plants (Camellia sinensis L.). Int. J. Mol. Sci. 2018, 19, 3938. [CrossRef] [PubMed]

53. Lu, Y.; He, Y.; Zhu, S.; Zhong, X.; Chen, D.; Liu, Z. New Acylglycosides Flavones from Fuzhuan Brick Tea and Simulation Analysis of Their Bioactive Effects. Int. J. Mol. Sci. 2019, 20, 494. [CrossRef] [PubMed]

54. Nanjo, F.; Mori, M.; Goto, K.; Hara, Y. Radical scavenging activity of tea catechins and their related compounds. Biosci. Biotechnol. Biochem. 1999, 63, 1621-1623. [CrossRef] [PubMed]

55. Masek, A.; Chrzescijanska, E.; Latos, M.; Zaborski, M.; Podsędek, A. Antioxidant and Antiradical Properties of Green Tea Extract Compounds. Int. J. Electrochem. 2017, 12, 6600-6610. [CrossRef]

56. He, J.; Xu, L.; Yang, L.; Wang, X. Epigallocatechin Gallate Is the Most Effective Catechin Against Antioxidant Stress via Hydrogen Peroxide and Radical Scavenging Activity. Med. Sci. Monit. 2018, 24, 8198-8206. [CrossRef] [PubMed]

57. Bartoszek, M.; Polak, J.; Chorażewski, M. Comparison of antioxidant capacities of different types of tea using the spectroscopy methods and semi-empirical mathematical model. Eur. Food Res. Technol. 2017, 244, 595-601. [CrossRef]

58. Lotito, S.B.; Fraga, C.G. Catechins delay lipid oxidation and alpha-tocopherol and beta-carotene depletion following ascorbate depletion in human plasma. Proc. Soc. Exp. Biol. Med. 2000, 225, 32-38. [CrossRef]

59. Metsalu, T.; Vilo, J. ClustVis: A web tool for visualizing clustering of multivariate data using Principal Component Analysis and heatmap. Nucleic Acids Res. 2015, 43, W566-W570. [CrossRef]

60. Pacifico, S.; Galasso, S.; Piccolella, S.; Kretschmer, N.; Pan, S.-P.; Nocera, P.; Lettieri, A.; Bauer, R.; Monaco, P. Winter wild fennel leaves as a source of anti-inflammatory and antioxidant polyphenols. Arab. J. Chem. 2018, 11, 513-524. [CrossRef]

61. Faramarzi, S.; Pacifico, S.; Yadollahi, A.; Lettieri, A.; Nocera, P.; Piccolella, S. Red-fleshed Apples: Old Autochthonous Fruits as a Novel Source of Anthocyanin Antioxidants. Plant Foods Hum. Nutr. 2015, 70, 324-330. [CrossRef] 
62. Pacifico, S.; Gallicchio, M.; Fiorentino, A.; Fischer, A.; Meyer, U.; Stintzing, F.C. Antioxidant properties and cytotoxic effects on human cancer cell lines of aqueous fermented and lipophilic quince (Cydonia oblonga Mill.) preparations. Food Chem. Toxicol. 2012, 50, 4130-4135. [CrossRef] [PubMed]

63. Sun, T.; Tanumihardjo, S.A. An Integrated Approach to Evaluate Food Antioxidant Capacity. J. Food Sci. 2007, 72, R159-R165. [CrossRef] [PubMed]

Sample Availability: Samples of the compounds are available from the authors.

(C) 2020 by the authors. Licensee MDPI, Basel, Switzerland. This article is an open access article distributed under the terms and conditions of the Creative Commons Attribution (CC BY) license (http://creativecommons.org/licenses/by/4.0/). 